\title{
Nusselt numbers for Poiseuille flow over isoflux parallel ridges accounting for meniscus curvature
}

\author{
Toby L. Kirk ${ }^{1} \dagger$, Marc Hodes ${ }^{2}$ and Demetrios T. Papageorgiou ${ }^{1}$ \\ ${ }^{1}$ Department of Mathematics, Imperial College London, London SW7 2AZ, UK \\ ${ }^{2}$ Department of Mechanical Engineering, Tufts University, Medford, MA 02155, USA
}

(Received xx; revised xx; accepted $\mathrm{xx}$ )

We investigate forced convection in a parallel plate-geometry microchannel with superhydrophobic walls consisting of a periodic array of ridges aligned parallel to the direction of a Poiseuille flow. In the de-wetted (Cassie) state, the liquid contacts the channel walls only at the tips of the ridges, where we apply a constant heat flux boundary condition. The subsequent hydrodynamic and thermal problems within the liquid are then analysed accounting for curvature of the liquid-gas interface (meniscus) using boundary perturbation, assuming a small deflection from flat. The effects of this surface deformation on both the effective hydrodynamic slip length and the Nusselt number are computed analytically in the form of eigenfunction expansions, reducing the problem to a set of dual series equations for the expansion coefficients which must, in general, be solved numerically. The Nusselt number quantifies the convective heat transfer, the results for which are completely captured in a single figure, presented as a function of channel geometry at each order in the perturbation. Asymptotic solutions for channel heights large compared to ridge period are compared to numerical solutions of the dual series. The asymptotic slip length expressions are shown to consist of only two terms, with all other terms exponentially small. As a result these expressions are accurate even for heights as low as half the ridge period, and hence useful for engineering applications.

Key words:

\section{Introduction}

The use of superhydrophobic surfaces has attracted the attention of many researchers in recent years, owing to their ability to reduce flow resistance in microfluidic applications. They are partially non-wetting surfaces consisting of micrometer or sub-micrometer roughness - typically a pattern of grooves, ridges or pillars - that resists a liquid fully wetting the solid substrate. It is possible for a liquid to assume a stable state (the CassieBaxter state) in which it only makes contact with the surface at the tips of the structures, and partially rides on a lubricating layer of vapor (and perhaps non-condensible gas) trapped in the cavities. This decrease in liquid-solid contact area results in a reduction in viscous drag, and the notion of apparent hydrodynamic slip.

A geometry of microstructures that has been extensively studied is a periodic array of rectangular ridges and grooves. Ou et al. (2004) and Ou \& Rothstein (2005) performed

$\dagger$ Email address for correspondence: tlk12@ic.ac.uk 
experiments in microchannels with ridges aligned parallel (longitudinal) to the flow direction, reporting up to $40 \%$ reductions in flow resistance. Experimental and numerical work by Davies et al. (2006) and Maynes et al. (2007) were conducted for microchannels with ridges aligned parallel and perpendicular (transverse) to the flow, respectively, with the greatest drag reduction found for lower Reynolds numbers. Analytical work for flow over such surfaces has been done earlier, however, by Philip (1972) and Lauga \& Stone (2003). Philip (1972) found solutions using conformal mapping theory for Stokes flow over a range of different geometries, including channel flow with longitudinal or transverse ridges on only one wall, and pipe flow with longitudinal ridges. Lauga \& Stone (2003) found analytical solutions for Stokes flow in a pipe patterned with transverse ridges, using separation of variables. This method was later extended by Teo \& Khoo (2009) to solve the remaining combinations of transverse/longitudinal ridges on one/both walls for pressure-driven channel flows. In addition to pressure-driven flows, shear-driven flows over ridged surfaces have also been studied (Cottin-Bizonne et al. 2004; Ybert et al. 2007; Priezjev et al. 2005).

Most of the aforementioned analytical and computational studies have assumed that the liquid-gas interfaces (menisci) are flat for simplicity (or for the case of pipe flow, that the curvature matched that of the pipe wall). However, the effect of meniscus curvature for parallel plate channels has also been investigated: analytically for unbounded shearflows over transverse ridges (Davis \& Lauga 2009) and longitudinal ridges (Crowdy 2010) for large solid fractions; perturbatively for small meniscus protrusion angles and arbitrary solid fractions by Sbragaglia \& Prosperetti (2007); and numerically for arbitrary angles and solid fractions by Teo \& Khoo (2010). The latter two studies considered both bounded and unbounded flows. Overall these studies showed that meniscus curvature can greatly inhibit or enhance the potential slip, depending on whether the interface protrudes into or out of the gas cavities, and the orientation of the ridges. Davis \& Lauga found that for transverse ridges that excessive protrusion out of the cavities can cause the slip length to become negative, but this does not occur for longitudinal ridges.

Flows over such surfaces in the presence of heat transfer have received much less attention, but are relevent and of practical and academic interest. The practical interest lies in, e.g., the thermal management of electronics via microchannel cooling of microprocessors whereby transfer of heat from the solid substrate (silicon) to a liquid coolant is desired, with as little thermal resistance as possible. A significant portion of the total thermal resistance is the caloric resistance of the coolant attributable to the energy required to increase its bulk temperature as it flows through the microchannel (Tuckerman \& Pease 1981; Hodes et al. 2014; Lam et al. 2015). The structured walls increase the mass flow rate, and thus decrease the caloric resistance. However, the Nusselt number $(N u)$, which characterises the heat transfer from solid to liquid due to convection, decreases owing to less solid-liquid contact area (Enright et al. 2006; Maynes et al. 2008, 2013; Maynes \& Crockett 2014). Thus, evaluation of the net effect of structuring on heat transfer requires evaluating the caloric and convective resistances, i.e., obtaining expressions for the slip length and $N u$ for various geometries and physical effects.

A small number of previous studies have considered thermal transport in channels with walls structured with parallel ridges. Enright et al. (2006) used an analytical model of the thermal transport in a parallel-plate channel with imposed hydrodynamic slip, neglecting the periodicity of the heating due to the ridge geometry. Maynes et al. (2008) used full numerical simulations to study the case of transverse ridges on both walls, which are held at a constant temperature, while also accounting for heat transfer through the gas cavities. The cases of constant heat flux through ridges oriented transverse and parallel to the flow direction have been studied by Maynes et al. (2013) and Maynes \& Crockett 
(2014), respectively. Longitudinal ridges were shown in each case to have greater potential for heat transfer enhancement, however the aforementioned study by Maynes \& Crockett did not solve for the velocity field but used a Navier slip approximation, and so neglected the potentially important variation in the velocity near the ridge structures - where the heat is entering (or leaving) the liquid. This effect is captured in the current study where we calculate the full velocity field, and show that the Navier slip assumption breaks down for geometries where the channel height is comparable to the ridge period. More recently, Enright et al. (2014) derived Nusselt number expressions in a channel assuming asymmetric hydrodynamic and thermal slip profiles (different slip lengths on opposing walls) imposing constant heat flux, and Cheng et al. (2015) investigated the effect of Reynolds number using full numerical simulations, imposing constant temperature. In addition to parallel ridges, both studies also considered structuring with pillars.

Many physical effects at the meniscus can be important to the heat transfer problem, including thermocapillary stresses, evaporation/condensation, and meniscus curvature. Thermocapillary stress for flow over ridged surfaces has been considered by Baier et al. (2010) and recently by Hodes et al. (2015a), and evaporation/condenstaion investigated by Hodes et al. (2015b). However, all previous work on heat transfer (including that mentioned in the previous paragraph) has assumed a flat meniscus. Very recently, Lam et al. (2016) investigated the effect of meniscus curvature on the thermal problem in a semi-infinite domain, but without convection and therefore interaction with the velocity field. To our knowledge, the effect of meniscus curvature on the convective thermal transport problem in a microchannel has not been considered. This is the primary goal of this paper. We will calculate the Nusselt number for channel flow over longitudinal ridges with constant heat flux, including small meniscus curvature via boundary perturbation. In doing so, for the hydrodynamic problem we will extend the small curvature analysis of Sbragaglia \& Prosperetti (2007), who considered longitudinal ridges on one channel wall, to the case of longitudinal ridges on both walls. Additionally, we will bridge the gap between the finite channel height solution, requiring a numerical solution of dual series equations, and the analytical semi-infinite shear-driven solution, to provide analytical expressions for the hydrodynamic slip length, accurate even for channel heights comparable to the ridge period. The perturbation will be performed on the full coupled velocity and thermal problems, and the solutions determined semi-analytically in terms of eigenfunction expansions. The resulting slip length and Nusselt number will then be presented as functions of channel geometry. The slip length is accurately described by the asymptotic expressions (6.4), (6.10), (6.11), whereas the Nusselt number results are completely captured in a single graph, figure $11(b)$.

In section 2 we formulate both the hydrodynamic and thermal problems, and the shape and description of the meniscus. Section 3 describes the boundary perturbation method for small curvature, and the resulting mathematical problems at each order. Section 4 details the solutions for the velocity problems, and section 5 the solutions for the temperature problems, with the asymptotic solutions for large channel heights in section 6 . The results are then presented in section 7 , and conclusions in section 8 .

\section{Formulation}

\subsection{Governing equations}

We consider hydrodynamically and thermally fully-developed flow in a parallel plate channel, with uniformly spaced parallel ridges aligned with the flow direction, as in figure 1. The top and bottom walls are structured identically, so that the flow is symmetric 


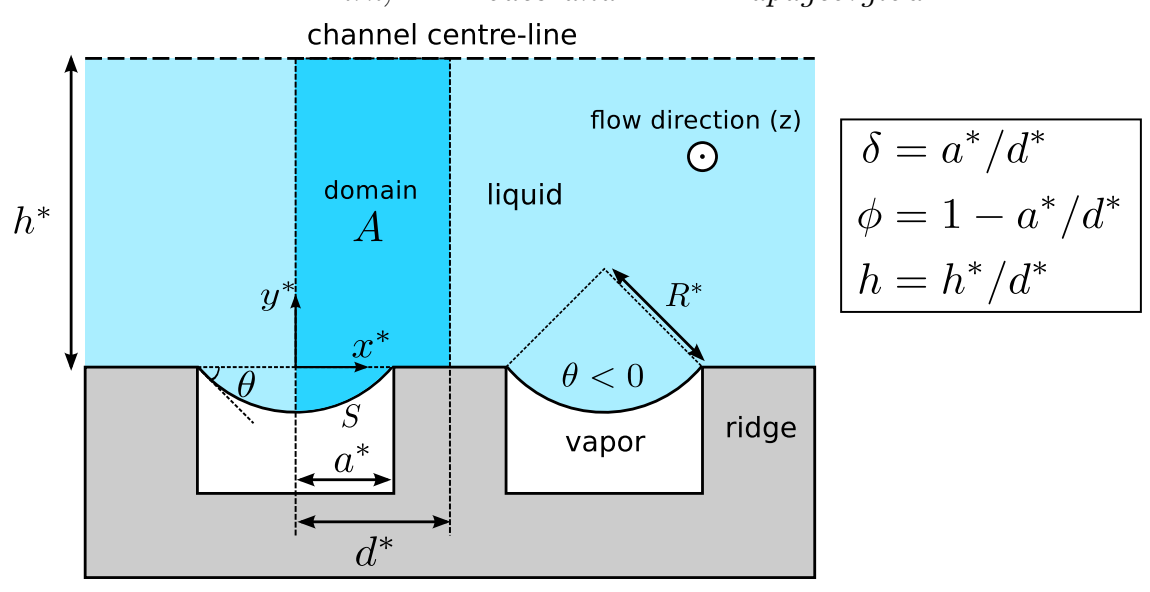

Figure 1. Schematic of the flow configuration, showing the liquid-solid contact and groove dimensions. Domain $A$ is the cross-section used for the analysis.

about the centre plane of the channel. Surface tension maintains the liquid in the Cassie state, i.e., the liquid contacts the solid substrate only on the tops of the ridges. The grooves between ridges are filled with vapor (and perhaps also non-condensible gas). The pressure difference between the liquid and gas causes the liquid-gas interfaces (menisci) to protrude into (or out of) the cavities. The protrusion is considered small enough that the liquid-solid contact angle is small enough for the contact line to stay pinned at the corners of the ridges.

The period of the ridges is denoted by $2 d^{*}$, the width of each groove by $2 a^{*}$, and the distance from the top of the ridges to the channel centre-line by $h^{*}$, as shown in figure 1. The use of an asterisk indicates a dimensional quantity. The meniscus protrudes at an angle $\theta$ relative to the horizontal at the triple (liquid-solid-gas) contact lines. Positive $\theta$ corresponds to protrusion out of the groove. The $x^{*}, y^{*}$ and $z^{*}$ directions are taken to be the spanwise, transverse (wall-normal), and streamwise directions, respectively, with $x^{*}$ and $y^{*}$ measured from the midpoint between the top of two adjacent ridges. Assuming the number of ridges to be sufficiently large, we can consider the flow to be periodic in the spanwise $\left(x^{*}\right)$ direction, with the same period as the ridges, $2 d^{*}$. Hence, we only need to consider a single period window, from the centre of one ridge to the next, say $\left|x^{*}\right| \leqslant d^{*}$. In addition, symmetry about the line $x^{*}=0$ means we can further restrict attention to just half of a period, $0 \leqslant x^{*} \leqslant d^{*}$. The appropriate flow domain to consider for analysis, $A$, is shown in figure 1, with its bottom boundary consisting of half of one liquid-solid interface and half of one curved meniscus interface, $S$. Lengths are nondimensionalised with respect to the width of this domain, $d^{*}$, and we introduce the solid fraction, $\phi=1-a^{*} / d^{*}$, and the complementary cavity fraction, $\delta=a^{*} / d^{*}$. The dimensionless (half) channel height is defined as $h=h^{*} / d^{*}$. Neglecting thermocapillary stress-induced convection and evapouration/condensation at the meniscus, there will be no transverse or spanwise flow in fully-developed conditions; only the streamwise velocity component $w^{*}$ will be nonzero. We nondimensionalise $w^{*}$ by $\left(d^{* 2} / \mu\right)\left(-\mathrm{d} p^{*} / \mathrm{d} z^{*}\right)$, a velocity scale based on the prescribed streamwise pressure gradient $-\mathrm{d} p^{*} / \mathrm{d} z^{*}$, giving

$$
w=\frac{\mu}{\left(d^{*}\right)^{2}\left(-\mathrm{d} p^{*} / \mathrm{d} z^{*}\right)} w^{*},
$$

where $\mu$ is the dynamic viscosity of the liquid. Then, throughout the domain $w$ satisfies

$$
\nabla^{2} w=-1
$$


where $\nabla^{2}=\partial^{2} / \partial x^{2}+\partial^{2} / \partial y^{2}$ is the two-dimensional Laplacian. Symmetry across the left $(x=0)$, right $(x=1)$, and top $(y=h)$ boundaries of the domain $A$ translate to the conditions

$$
\frac{\partial w}{\partial x}(0, y)=0, \quad \frac{\partial w}{\partial x}(1, y)=0, \quad \frac{\partial w}{\partial y}(x, h)=0 .
$$

On the solid ridge we apply the no-slip condition,

$$
w(x, 0)=0 \quad \text { for } \delta<x \leqslant 1,
$$

while on the meniscus $S$ we assume no tangential stress,

$$
\boldsymbol{n} \cdot \boldsymbol{\nabla} w=0 \quad \text { on } S,
$$

where $\boldsymbol{n}$ is the unit (outward) normal to the interface. The domain, equations and boundary conditions for the $w$ (and $T$ ) problem are summarised in figure 2 .

In the thermal problem for the temperature field $T^{*}$, we assume that the only source of heating of the fluid is through its contact with the solid ridges, where it experiences a constant heat flux per unit area, $q_{s l}^{\prime \prime}$. Specifically, on the solid-liquid interface we have

$$
-k \frac{\partial T^{*}}{\partial y^{*}}=q_{s l}^{\prime \prime} \quad \text { for } \delta<x \leqslant 1
$$

The numerical results of Maynes et al. (2008) show that, for a flat meniscus, the rate of heat conducting into the liquid through the gas cavities is negligible in comparison to the rate entering through the solid ridges for cavity depths greater than about $25 \%$ of the cavity width. We will assume this here, and take the gas phase to be adiabatic, applying a zero heat flux condition on the meniscus:

$$
\boldsymbol{n} \cdot \boldsymbol{\nabla} T^{*}=0 \quad \text { on } S .
$$

Viscous dissipation, Marangoni induced convection, and evaporation and condensation at the meniscus, are neglected. The dimensional thermal energy equation is then

$$
w^{*} \frac{\partial T^{*}}{\partial z^{*}}=\alpha\left(\frac{\partial^{2} T^{*}}{\partial x^{* 2}}+\frac{\partial^{2} T^{*}}{\partial y^{* 2}}+\frac{\partial^{2} T^{*}}{\partial z^{* 2}}\right),
$$

where $\alpha=k /\left(\rho c_{p}\right)$ is the thermal diffusivity of the liquid, $k$ is the thermal conductivity, $\rho$ is the density, and $c_{p}$ is the specific heat, all assumed constant. The thermally fullydeveloped assumption, i.e., that we are sufficiently far from the channel inlet so as to neglect entrance effects, is characterised by the self-similarity property (Bergman et al. 2011):

$$
\frac{\partial}{\partial z^{*}}\left[\frac{T^{*}\left(x^{*}, y^{*}, z^{*}\right)-T_{s l}^{*}\left(x^{*}, z^{*}\right)}{T_{m}^{*}\left(z^{*}\right)-T_{s l}^{*}\left(x^{*}, z^{*}\right)}\right]=0,
$$

where $T_{s l}^{*}$ is the temperature on the solid-liquid interface, and $T_{m}^{*}$ is the mixed-mean liquid temperature,

$$
T_{m}^{*}=\frac{\int_{A} w^{*} T^{*} \mathrm{~d} x^{*} \mathrm{~d} y^{*}}{\int_{A} w^{*} \mathrm{~d} x^{*} \mathrm{~d} y^{*}} .
$$

For the constant heat flux boundary condition (2.6), the property (2.9) can be shown, conveniently, to imply $\partial T^{*} / \partial z^{*}=\mathrm{d} T_{m}^{*} / \mathrm{d} z^{*}=$ constant. As a consequence, the axial conduction term, $\partial^{2} T^{*} / \partial z^{* 2}$ is exactly zero and can be dropped from (2.8). The constant can be found by considering an energy balance on a control volume of cross-section $A$ and depth $\mathrm{d} z^{*}$, yielding

$$
\frac{\partial T^{*}}{\partial z^{*}}=\frac{q_{s l}^{\prime \prime}\left(d^{*}-a^{*}\right)}{\rho Q^{*} c_{p}},
$$




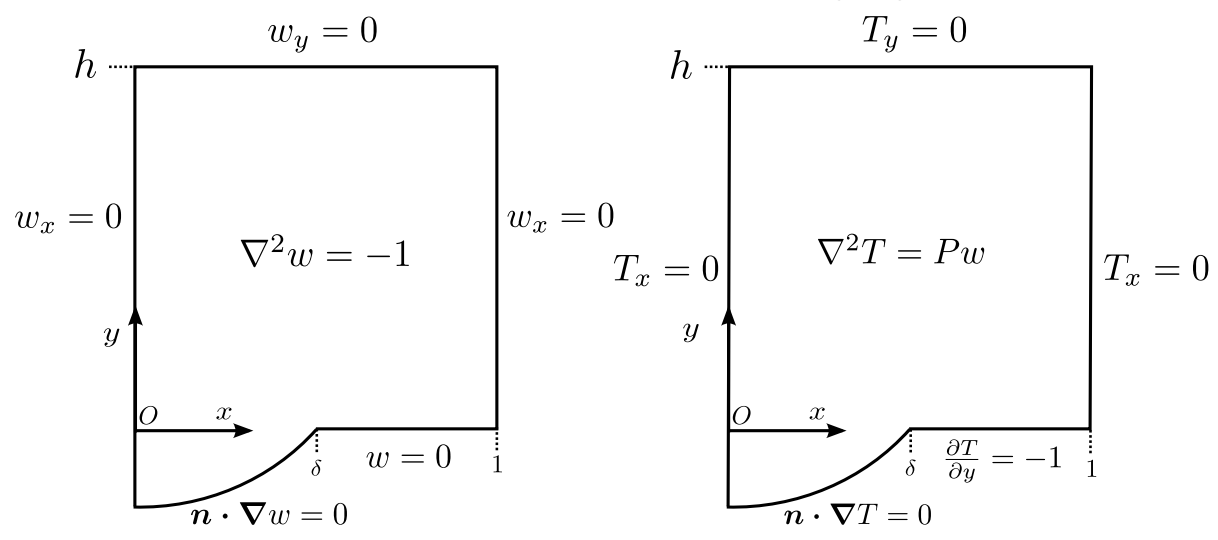

Figure 2. Domains, governing equations, and boundary conditions for the $w$ and $T$ problems. Subscripts denote partial differentiation.

where $Q^{*}$ is the total volume flow rate through the cross-section $A$.

We nondimensionalise temperature by subtracting $T_{m}^{*}$ and then scaling by the characteristic temperature scale, $\Delta T^{*}=q_{s l}^{\prime \prime} d^{*} / k$, from the heat flux condition (2.6), to give

$$
T=\frac{T^{*}-T_{m}^{*}}{\Delta T^{*}}=\frac{k\left(T^{*}-T_{m}^{*}\right)}{q_{s l}^{\prime \prime} d^{*}} .
$$

Then the dimensionless form of the thermal energy equation (2.8) is

$$
P w=\nabla^{2} T
$$

where it is convenient to introduce

$$
P=\frac{\phi}{Q}
$$

with $Q$ the nondimensional flow rate. Thus the forcing $P w$ is determined completely by the hydrodynamic problem.

Just as with the velocity, the left $(x=0)$, right $(x=1)$ and top $(y=h)$ boundaries of the domain are symmetry boundaries of $T$, and so

$$
\frac{\partial T}{\partial x}(0, y)=0, \quad \frac{\partial T}{\partial x}(1, y)=0, \quad \frac{\partial T}{\partial y}(x, h)=0 .
$$

The constant heat flux condition (2.6) on the ridge becomes

$$
\frac{\partial T}{\partial y}=-1 \quad \text { for } \delta<x \leqslant 1,
$$

and the adiabatic condition on the meniscus (2.7) is unchanged. Just as for the $w$ problem, see figure 2 for a summary of the problem for $T$. The $w$ problem can be solved in isolation, and then the solution $w$ appears as a forcing in the Poisson problem for $T$. Note that this is possible for a meniscus of any shape or curvature.

The quantity that characterises the heat transfer problem is the Nusselt number, $N u$, which captures the influence of the structured surface (including apparent slip and periodic heating) on the overall thermal transport due to convection. We follow Maynes \& Crockett (2014) and define a local Nusselt number

$$
N u(x)=\frac{h_{T}(x) D}{k},
$$


where $D$ is the hydraulic diameter, $D=4 h^{*}$, and $h_{T}(x)$ is the local heat transfer coefficient (not to be confused with $h$, the nondimensional half channel thickness), defined as $h_{T}(x)=q_{s l}^{\prime \prime} /\left(T_{s l}^{*}-T_{m}^{*}\right)$ on the solid (where the temperature is $\left.T_{s l}^{*}\right)$, and zero on the meniscus. Hence

$$
N u(x)= \begin{cases}\frac{4 h}{T_{s l}(x)}, & \text { on solid, } \delta<x \leqslant 1 \\ 0, & \text { on meniscus, } 0 \leqslant x<\delta\end{cases}
$$

Note that $T_{s l}(x)$ is not a function of $z$, which can be shown from the nondimensionalisation (2.12) and fully-developed assumption (2.9). Averaging $N u(x)$ over the composite interface (solid-liquid interface and meniscus) gives the average Nusselt number characterising the structured surface,

$$
\overline{N u}=\frac{1}{\phi+|S|} \int_{\delta}^{1} N u(x) \mathrm{d} x,
$$

where $\phi+|S|$ is the total length of the composite interface. This length factor will have no effect on the results in this paper, however (see (3.25)). We remark that there are other ways of defining a Nusselt number to represent the structured surface, such as using only the temperature at the centre of the ridge, where it will be highest.

\subsection{Meniscus curvature}

We will take a perturbative approach to investigate the effect of meniscus curvature on the heat transfer problem, based on the assumption that the deflection of the interface relative to a flat one is small. As in Sbragaglia \& Prosperetti (2007), we represent the position of the meniscus in the form

$$
y=-\epsilon \eta(x), \quad 0 \leqslant x \leqslant \delta,
$$

where $\eta(x)$ is some shape function and $\epsilon$ is the parameter that is taken to be small compared to 1. To relate $\epsilon$ to dimensional quantities and find an expression for $\eta(x)$, we can briefly return to dimensional variables and invoke the Young-Laplace equation relating the capillary pressure difference across a static interface to its radius of curvature. Denoting the pressure on the liquid side of the interface by $p_{l}^{*}$, that on the gas side by $p_{g}^{*}$, and the local radius of curvature by $R^{*}$,

$$
p_{l}^{*}-p_{g}^{*}=\frac{\sigma}{R^{*}}
$$

where $\sigma$ is the surface tension along the meniscus. As there is no flow in the transverse directions, $p_{l}^{*}$ is constant throughout any given flow cross-section, and we have neglected the gas phase so will assume $p_{g}^{*}$ is constant throughout the length of the cavity. Hence, for a given value of $z^{*}$, the difference $p_{l}^{*}-p_{g}^{*}$ is constant along the interface, giving a constant radius of curvature. Assuming a small streamwise capillary number, as we move down the channel, the pressure $p_{l}^{*}$ will fall and $R^{*}$ will increase, but the change will be slow enough not to invalidate the assumption of locally unidirectional flow ( $\mathrm{Ou}$ et al. 2004; Ou \& Rothstein 2005).

If $p_{l}^{*}>p_{g}^{*}$ then $R^{*}>0$ and the meniscus protudes into the cavity (negative protrusion angle $\theta$ ), and if $p_{l}^{*}<p_{g}^{*}$ then $R^{*}<0$ which we can interpret as the meniscus protruding upwards into the liquid (positive protrusion angle $\theta$ ). In general, both signs of $\theta$ are possible since the Young equilibrium angle can lie anywhere between the advancing and receding contact angles. 
For protrusion into the cavity, a circle of radius $R^{*}$ passing through the corners of neighbouring ridges, i.e., $x^{*}= \pm a^{*}$, is defined by

$$
y^{*}=\sqrt{R^{* 2}-a^{* 2}}-\sqrt{R^{* 2}-x^{* 2}} .
$$

Defining $R=R^{*} / d^{*}$, in nondimensional form this is

$$
y=\sqrt{R^{2}-\delta^{2}}-\sqrt{R^{2}-x^{2}},
$$

which when expanded for $R \gg 1$ gives

$$
y=-\frac{1}{2 R}\left(\delta^{2}-x^{2}\right)+O\left(\frac{1}{R^{3}}\right) .
$$

Thus we define our small parameter as $\epsilon=1 /(2 R)$ and shape function as $\eta(x)=\delta^{2}-x^{2}$. Then, for $\epsilon \ll 1$, the meniscus is prescribed by

$$
y=-\epsilon \eta(x)+O\left(\epsilon^{3}\right) .
$$

As we will not calculate up to third-order in $\epsilon$ in our perturbative expansion, only firstorder, the leading order approximation $y=-\epsilon \eta$ will be sufficient. The case of protrusion into the liquid corresponds to negative $\epsilon$. The relation between $\epsilon$ and the protrusion angle $\theta$ is found from geometry,

$$
\epsilon=\frac{1}{2 R}=-\frac{\sin \theta}{2 \delta}
$$

Water on low surface energy fluoropolymer has a maximum advancing contact angle of approximately $110^{\circ}$ (measured from vertical), corresponding to a maximum (negative) protrusion angle of $\theta=-20^{\circ}$. For low solid fractions, e.g., $\phi=1-\delta=0.01$, this gives a maximum value of $\epsilon=0.17$.

\section{Boundary perturbation}

The method of analysis we will employ is boundary perturbation, whereby the solution to the problem with a curved meniscus (with small curvature characterised by $|\epsilon| \ll 1$ ) is approximated by a hierarchy of problems in powers of $\epsilon$, each with a flat meniscus. We base the method on that of Sbragaglia \& Prosperetti (2007) who solved the purely hydrodynamic problem for a similar geometry. If the shape and position of the meniscus is written in the form (with $O\left(\epsilon^{3}\right)$ error)

$$
y=-\epsilon \eta(x), \quad 0 \leqslant x \leqslant \delta,
$$

then the vanishing normal derivative condition on the meniscus (2.5) can be written

$$
\epsilon \frac{\mathrm{d} \eta}{\mathrm{d} x} \frac{\partial w}{\partial x}+\frac{\partial w}{\partial y}=0 \quad \text { on } y=-\epsilon \eta .
$$

Since $|\epsilon| \ll 1$, we Taylor expand the quantities evaluated at $y=-\epsilon \eta$ about $y=0$, assuming $w$ is sufficiently smooth. Taylor expanding $w$ we have

$$
w(x,-\epsilon \eta)=w(x, 0)-\epsilon \eta \frac{\partial w}{\partial y}(x, 0)+O\left(\epsilon^{2}\right),
$$


and in particular,

$$
\begin{aligned}
& \frac{\partial w}{\partial y}(x,-\epsilon \eta)=\frac{\partial w}{\partial y}(x, 0)-\epsilon \eta \frac{\partial^{2} w}{\partial y^{2}}(x, 0)+O\left(\epsilon^{2}\right), \\
& \frac{\partial w}{\partial x}(x,-\epsilon \eta)=\frac{\partial w}{\partial x}(x, 0)+O(\epsilon) .
\end{aligned}
$$

Substitution into (3.2) gives, up to terms of $O(\epsilon)$,

$$
\frac{\partial w}{\partial y}(x, 0)-\epsilon \eta \frac{\partial^{2} w}{\partial y^{2}}(x, 0)+\epsilon \frac{\mathrm{d} \eta}{\mathrm{d} x} \frac{\partial w}{\partial x}(x, 0)+O\left(\epsilon^{2}\right)=0 .
$$

Then substituting a regular perturbative expansion of $w$ in powers of $\epsilon$,

$$
w=w^{(0)}+\epsilon w^{(1)}+O\left(\epsilon^{2}\right),
$$

and equating terms at each order of $\epsilon$ will yield a series of problems for $w^{(0)}, w^{(1)}, \ldots$, each with an interfacial boundary condition now at $y=0$. At $O\left(\epsilon^{0}\right)$, equation (3.6) gives

$$
\partial_{y} w^{(0)}(x, 0)=0 \quad \text { for } 0 \leqslant x<\delta,
$$

and at $O\left(\epsilon^{1}\right)$,

$$
\partial_{y} w^{(1)}(x, 0)=\eta \partial_{y y} w^{(0)}(x, 0)-\eta^{\prime} \partial_{x} w^{(0)}(x, 0) \text { for } 0 \leqslant x<\delta,
$$

where for brevity we denote $\partial_{y}=\partial / \partial y, \partial_{y y}=\partial^{2} / \partial y^{2}$ and $\eta^{\prime}=\mathrm{d} \eta / \mathrm{d} x$. The governing equation, (2.2), gives

$$
\nabla^{2} w^{(0)}=-1
$$

at $O\left(\epsilon^{0}\right)$ and

$$
\nabla^{2} w^{(1)}=0
$$

at $O\left(\epsilon^{1}\right)$ (and at all higher orders). The no-slip condition on the solid ridge (2.4) and the symmetry conditions (2.3) are satisfied at all orders, and thus by $w^{(0)}$ and $w^{(1)}$.

The boundary perturbation analysis for the temperature field $T$, is similar to that for the velocity field, and expressed as

$$
T=T^{(0)}+\epsilon T^{(1)}+O\left(\epsilon^{2}\right) .
$$

The meniscus condition (2.7) is identical to that for $w$, thus

$$
\begin{array}{ll}
\partial_{y} T^{(0)}(x, 0)=0, & 0 \leqslant x<\delta, \\
\partial_{y} T^{(1)}(x, 0)=\eta \partial_{y y} T^{(0)}(x, 0)-\eta^{\prime} \partial_{x} T^{(0)}(x, 0), & 0 \leqslant x<\delta,
\end{array}
$$

and the symmetry boundary conditions (2.15) are satisfied at all orders. Only the boundary condition on the ridge and the governing equation differ from those of the velocity problem. The constant heat flux boundary condition on ridge, $\partial T / \partial y=-1$, gives

$$
\begin{aligned}
& \partial_{y} T^{(0)}=-1, \quad \delta<x \leqslant 1, \\
& \partial_{y} T^{(1)}=0, \quad \delta<x \leqslant 1 .
\end{aligned}
$$

Recall that $T$ satisfies Poisson's equation (2.13) with forcing $P w$, where $P=\phi / Q$. Hence we must expand the flow rate $Q$. When $\epsilon>0$ (meniscus protrudes into the cavity)

$$
\begin{aligned}
Q & =\int_{0}^{1} \int_{0}^{h} w(x, y ; \epsilon) \mathrm{d} y \mathrm{~d} x+\int_{0}^{\delta} \int_{-\epsilon \eta}^{0} w(x, y ; \epsilon) \mathrm{d} y \mathrm{~d} x, \\
& =Q^{(0)}+\epsilon\left(Q_{1}^{(1)}+Q_{2}^{(1)}\right)+O\left(\epsilon^{2}\right),
\end{aligned}
$$




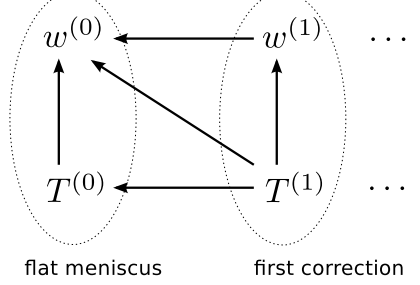

FiguRE 3. Schematic of the four fields to be found. The arrows denote dependencies, i.e., "is forced by."

where

$$
\begin{aligned}
& Q^{(0)}=\int_{0}^{h} \int_{0}^{1} w^{(0)}(x, y) \mathrm{d} x \mathrm{~d} y, \\
& Q_{1}^{(1)}=\int_{0}^{h} \int_{0}^{1} w^{(1)}(x, y) \mathrm{d} x \mathrm{~d} y, \\
& Q_{2}^{(1)}=\int_{0}^{\delta} w^{(0)}(x, 0) \eta(x) \mathrm{d} x .
\end{aligned}
$$

If $\epsilon<0$, the integral definition of $Q$ cannot be split up as in (3.17), but the resulting expansion (3.18) is the same. Thus (3.18) holds for both positive and negative $\epsilon$. We can interpret $Q_{1}^{(1)}$ as the $O(\epsilon)$ contribution to the flow rate due to the change in the velocity field, and $Q_{2}^{(1)}$ as the $O(\epsilon)$ contribution due to the change in cross-sectional area of the domain. Then, the expansion $P=P^{(0)}+\epsilon P^{(1)}+O\left(\epsilon^{2}\right)$ of $P=\phi / Q$ up to $O(\epsilon)$ is given by

$$
P^{(0)}=\frac{\phi}{Q^{(0)}}, \quad P^{(1)}=-P^{(0)} \frac{Q_{1}^{(1)}+Q_{2}^{(1)}}{Q^{(0)}} .
$$

Substituting the expansions for $T, w$, and $P$ into $\nabla^{2} T=P w$ gives

$$
\begin{aligned}
& \nabla^{2} T^{(0)}=P^{(0)} w^{(0)} \quad \text { at } O\left(\epsilon^{0}\right) \\
& \nabla^{2} T^{(1)}=P^{(0)} w^{(1)}+P^{(1)} w^{(0)} \quad \text { at } O\left(\epsilon^{1}\right) \text {. }
\end{aligned}
$$

Finally, expanding $\overline{N u}=\overline{N u}^{(0)}+\epsilon \overline{N u}^{(1)}+O\left(\epsilon^{2}\right)$,

$$
\overline{N u}^{(0)}=\int_{\delta}^{1} \frac{4 h}{T_{s}^{(0)}} \mathrm{d} x, \quad \overline{N u}^{(1)}=-\int_{\delta}^{1} \frac{4 h T_{s}^{(1)}}{\left(T_{s}^{(0)}\right)^{2}} \mathrm{~d} x .
$$

The length of the composite interface, i.e., the factor of $\phi+|S|$ in (2.19), is simply $1+O\left(\epsilon^{2}\right)$, so the increase in length is an $O\left(\epsilon^{2}\right)$ effect and does not affect the analysis in this paper.

The four fields and their dependencies on each other are shown in figure 3 . We will solve for them in the following order. The velocity problem can be solved independently of the temperature problem, and hence we will solve it first, for $w^{(0)}$ and then for $w^{(1)}$. Knowledge of $w^{(0)}$ allows us to solve for $T^{(0)}$, and finally knowledge of $w^{(0)}, w^{(1)}$, and $T^{(0)}$ is needed to solve for $T^{(1)}$. 


\section{Velocity problem, $w$}

The velocity problem considered here is similar to the one solved by Sbragaglia \& Prosperetti (2007). However, whereas they considered the case of longitudinal ridges on one channel wall and a flat plate with the no-slip boundary condition on the other, we are considering the symmetric case of ridges on both channel walls. Mathematically, this means we have the symmetry condition $\partial_{y} w=0$ instead of $w=0$ at the top $(y=h)$ of our domain. The cases will coincide in the limit of a semi-infinite channel, $h \rightarrow \infty$.

\subsection{Zeroth-order solution, $w^{(0)}$}

The problem for $w^{(0)}$ is

$$
\begin{aligned}
\nabla^{2} w^{(0)} & =-1, & & 0<x<1, \quad 0<y<h, \\
\partial_{y} w^{(0)}(x, 0) & =0, & & 0 \leqslant x<\delta, \\
w^{(0)}(x, 0) & =0, & & \delta<x \leqslant 1,
\end{aligned}
$$

along with vanishing normal derivatives on the remaining boundaries,

$$
\partial_{x} w^{(0)}(0, y)=\partial_{x} w^{(0)}(1, y)=\partial_{y} w^{(0)}(x, h)=0 .
$$

We will solve for $w^{(0)}$ in the form of an eigenfunction expansion, and the solution is provided by, e.g., Teo \& Khoo (2009). We write the solution as the one-dimensional flow profile (satisfying Poisson's equation and no-slip on $y=0$ ) and a perturbation part:

$$
w^{(0)}=-\frac{1}{2} y^{2}+h y+h \tilde{w}^{(0)} .
$$

Then $\tilde{w}^{(0)}$ satisfies Laplace's equation, with a constant shear stress on the flat meniscus,

$$
\begin{aligned}
\nabla^{2} \tilde{w}^{(0)} & =0, & & 0<x<1, \quad 0<y<h, \\
\partial_{y} \tilde{w}^{(0)}(x, 0) & =-1, & & 0 \leqslant x<\delta, \\
\tilde{w}^{(0)}(x, 0) & =0, & & \delta<x \leqslant 1,
\end{aligned}
$$

along with the symmetry conditions (4.4). Using separation of variables, or noting that $\tilde{w}^{(0)}$ is even in $x$ with period 2 , the solution can be written as a Fourier cosine series in $x$,

$$
\tilde{w}^{(0)}(x, y)=a_{0}^{(0)}+\sum_{n=1}^{\infty} a_{n}^{(0)} \frac{\cosh \left[k_{n}(y-h)\right]}{\cosh \left(k_{n} h\right)} \cos \left(k_{n} x\right),
$$

where $k_{n}=n \pi$. Note that this satisfies the requirement that $\partial_{y} \tilde{w}^{(0)}=0$ at $y=h$. The coefficients, $a_{n}^{(0)}$, are then determined from the conditions on the composite interface, (4.7) and (4.8), which lead to the dual series equations

$$
\begin{aligned}
a_{0}^{(0)}+\sum_{n=1}^{\infty} a_{n}^{(0)} \cos \left(k_{n} x\right) & =0, \quad \delta<x<1, \\
\sum_{n=1}^{\infty} a_{n}^{(0)} k_{n} \tanh \left(k_{n} h\right) \cos \left(k_{n} x\right) & =1, \quad 0<x<\delta .
\end{aligned}
$$

In the limit of a channel of infinite height, $h \rightarrow \infty$, these can be solved analytically (see section 6), but for a finite channel they must be solved numerically. The numerical method used is the same as that of previous authors (Lauga \& Stone 2003; Sbragaglia \& Prosperetti 2007; Teo \& Khoo 2009), and is outlined in an appendix of Sbragaglia 
\& Prosperetti (2007). Briefly, the method is to truncate each series at $n=N$, and then project each equation, in the region they hold, onto the eigenfunctions $\left\{\cos \left(k_{n} x\right)\right.$ : $0 \leqslant n \leqslant N\}$ to form an $(N+1)$-dimensional linear algebra problem for $\boldsymbol{a}^{(0)}=$ $\left(a_{0}^{(0)}, a_{1}^{(0)}, \ldots, a_{N}^{(0)}\right)^{T} \in \mathbb{R}^{N+1}$, written as $\boldsymbol{M} \boldsymbol{a}=\boldsymbol{f}$. The series structure (left hand sides) and the forcing (the right hand sides) of (4.10)-(4.11) determines $\boldsymbol{M} \in \mathbb{R}^{N+1} \times \mathbb{R}^{N+1}$ and $\boldsymbol{f} \in \mathbb{R}^{N+1}$, respectively.

From this solution, the leading order flow rate $Q^{(0)}$ is found by integrating (4.5) over the rectangular domain, giving

$$
Q^{(0)}=\frac{1}{3} h^{3}+h^{2} a_{0}^{(0)},
$$

and hence

$$
P^{(0)}=\frac{\phi}{\frac{1}{3} h^{3}+h^{2} a_{0}^{(0)}} .
$$

Lastly, from differentiating the series solution (4.9) term by term,

$$
\begin{aligned}
\partial_{y y} \tilde{w}^{(0)}(x, 0) & =\sum_{n=1}^{\infty} a_{n}^{(0)} k_{n}^{2} \cos \left(k_{n} x\right) \\
& =-\partial_{x x} \tilde{w}^{(0)}(x, 0) .
\end{aligned}
$$

Hence, $\nabla^{2} \tilde{w}^{(0)}=0$ and (via (4.5)) $\nabla^{2} w^{(0)}=-1$ are satisfied on the meniscus $y=0$ as well as in interior of the domain. This justifies simplifying the meniscus boundary condition (3.9) for $w^{(1)}$ in the manner of Sbragaglia \& Prosperetti (2007) to give condition (4.16) below.

\subsection{First-order solution, $w^{(1)}$}

The problem for the first-order perturbation $w^{(1)}$ is

$$
\begin{aligned}
\nabla^{2} w^{(1)} & =0, & & 0<x<1, \quad 0<y<h \\
\partial_{y} w^{(1)}(x, 0) & =-\partial_{x}\left[\eta \partial_{x} w^{(0)}(x, 0)\right]-\eta, & & 0 \leqslant x<\delta, \\
w^{(1)}(x, 0) & =0, & & \delta<x \leqslant 1,
\end{aligned}
$$

along with vanishing normal derivatives on the remaining boundaries,

$$
\partial_{x} w^{(1)}(0, y)=\partial_{x} w^{(1)}(1, y)=\partial_{y} w^{(1)}(x, h)=0 .
$$

We write the solution in a similar form to $w^{(0)}$, but a one-dimensional base profile is unnecessary, i.e., $w^{(1)}=h \tilde{w}^{(1)}$. Then, $\tilde{w}^{(1)}$ can also be written as a Fourier series:

$$
\tilde{w}^{(1)}(x, y)=a_{0}^{(1)}+\sum_{n=1}^{\infty} a_{n}^{(1)} \frac{\cosh \left[k_{n}(y-h)\right]}{\cosh \left(k_{n} h\right)} \cos \left(k_{n} x\right) .
$$

The no-slip condition (4.17) and interfacial condition (4.16) give another set of dual series equations:

$$
\begin{aligned}
a_{0}^{(1)}+\sum_{n=1}^{\infty} a_{n}^{(1)} \cos \left(k_{n} x\right) & =0, & \delta<x<1, \\
\sum_{n=1}^{\infty} a_{n}^{(1)} k_{n} \tanh \left(k_{n} h\right) \cos \left(k_{n} x\right)=\partial_{x}\left(\eta \partial_{x} \tilde{w}^{(0)}(x, 0)\right)+\frac{\eta}{h}, & & 0<x<\delta .
\end{aligned}
$$


This system for $a_{n}^{(1)}$ takes the same form as for $a_{n}^{(0)}$, with the only difference being the forcing on the right hand side of the second equation (4.21). Again, this can be solved analytically in the limit, $h \rightarrow \infty$ (see section 6 ), but for finite $h$ we must solve this numerically.

Once $a_{n}^{(0)}$ and $a_{n}^{(1)}$ are computed, the changes to the flow rate, $Q_{1}^{(1)}$ and $Q_{2}^{(1)}$, can be calculated by inserting the series (4.19) and (4.9) into (4.22) and (4.23), respectively. Then,

$$
\begin{aligned}
& Q_{1}^{(1)}=h^{2} a_{0}^{(1)}, \\
& Q_{2}^{(1)}=\frac{2}{3} \delta^{3} h a_{0}^{(0)}+h \sum_{n=1}^{\infty} a_{n}^{(0)} \int_{0}^{\delta} \eta(x) \cos k_{n} x \mathrm{~d} x,
\end{aligned}
$$

where each integral in the last sum can be calculated using integration by parts and $\eta(x)=\delta^{2}-x^{2}$ to give

$$
\int_{0}^{\delta} \eta(x) \cos k_{n} x \mathrm{~d} x=\frac{2 \sin k_{n} \delta}{k_{n}^{3}}-\frac{2 \delta \cos k_{n} \delta}{k_{n}^{2}} .
$$

We can define an apparent slip length by equating the flow rate $Q$ with what it would be, $Q_{\text {slip }}$, if the ridges were replaced with a flat surface subjected to a Navier slip condition, $w_{\text {slip }}(x, 0)=\lambda \partial_{y} w_{\text {slip }}(x, 0)$, from which we find

$$
Q_{\text {slip }}=\frac{1}{3} h^{3}+h^{2} \lambda .
$$

Here $\lambda$ is the slip length normalised by half the ridge period, i.e., $\lambda=\lambda^{*} / d^{*}$. We expand $\lambda$ similarly to $Q$,

$$
\lambda=\lambda^{(0)}+\epsilon\left(\lambda_{1}^{(1)}+\lambda_{2}^{(1)}\right)+O\left(\epsilon^{2}\right),
$$

where $\lambda^{(0)}, \lambda_{1}^{(1)}$, and $\lambda_{2}^{(1)}$ are the contributions from $Q^{(0)}, Q_{1}^{(1)}$, and $Q_{2}^{(1)}$, respectively. Thus, using (4.25), (4.12), (4.22) and (4.23), we find

$$
\begin{aligned}
& \lambda^{(0)}=a_{0}^{(0)} \\
& \lambda_{1}^{(1)}=a_{0}^{(1)} \\
& \lambda_{2}^{(1)}=\frac{2}{3} \frac{\delta^{3}}{h} a_{0}^{(0)}+\frac{1}{h} \sum_{n=1}^{\infty} a_{n}^{(0)}\left(\frac{2 \sin k_{n} \delta}{k_{n}^{3}}-\frac{2 \delta \cos k_{n} \delta}{k_{n}^{2}}\right) .
\end{aligned}
$$

\section{Temperature problem, $T$}

Now that we have determined the velocity field, $w=w^{(0)}+\epsilon w^{(1)}+O\left(\epsilon^{2}\right)$, up to firstorder, we can solve for the temperature field up to the same order, $T=T^{(0)}+\epsilon T^{(1)}+$ $O\left(\epsilon^{2}\right)$. Recall that the perturbation of the thermal problem and equating orders of $\epsilon$ was done at the beginning of section 3 , to give problems for $T^{(0)}$ and $T^{(1)}$, which we repeat below. The leading order thermal problem, corresponding to the case of a flat meniscus, has been solved by Maynes \& Crockett (2014) using a one-dimensional approximation of the velocity field, but not with the exact two-dimensional velocity field, as we will do here. The first-order problem for $T^{(1)}$, quantifying the effect of curvature on heat transfer in microchannels, has not to our knowledge been considered before, except very recently for the case of a semi-infinite $(h=\infty)$ domain with no advection $(w \equiv 0)$ (Lam et al. 2016). 


\subsection{Zeroth-order solution, $T^{(0)}$}

The problem for $T^{(0)}$, the temperature for the case of a flat meniscus, is given by (see $(2.13))$ :

$$
\begin{aligned}
\nabla^{2} T^{(0)} & =P^{(0)} w^{(0)}, & & 0<x<1, \quad 0<y<h \\
\partial_{y} T^{(0)}(x, 0) & =0, & & 0 \leqslant x<\delta, \\
\partial_{y} T^{(0)}(x, 0) & =-1, & & \delta<x \leqslant 1,
\end{aligned}
$$

along with vanishing normal derivatives on the remaining boundaries,

$$
\partial_{x} T^{(0)}(0, y)=\partial_{x} T^{(0)}(1, y)=\partial_{y} T^{(0)}(x, h)=0 .
$$

Recall that $P^{(0)}=\phi / Q^{(0)}=\phi /\left(\frac{1}{3} h^{3}+h^{2} a_{0}^{(0)}\right)$, and $w^{(0)}$ is given by (4.5), (4.9). Substituting $w^{(0)}$ into (5.1) gives

$$
\nabla^{2} T^{(0)}=P^{(0)}\left\{-\frac{1}{2} y^{2}+h y+h a_{0}^{(0)}+h \sum_{n=1}^{\infty} a_{n}^{(0)} \frac{\cosh \left[k_{n}(y-h)\right]}{\cosh \left(k_{n} h\right)} \cos \left(k_{n} x\right)\right\},
$$

and we see that the forcing consists of a quadratic in $y$ plus an infinite series, each term in the series depending on both $x$ and $y$. This can be handled by setting $T^{(0)}=$ $T_{p}^{(0)}+\widetilde{T}^{(0)}$, i.e., splitting it into a particular solution, $T_{p}^{(0)}$, that satisfies the forcing, and a remaining homogeneous (harmonic) part, $\widetilde{T}^{(0)}$, that enforces the boundary conditions on the composite interface, $y=0$. In addition, if $T_{p}^{(0)}$ satisfies the symmetry properties across $x=0, x=1$, and $y=h$, then $\widetilde{T}^{(0)}$ will also. To find such a $T_{p}^{(0)}$, satisfying $\nabla^{2} T_{p}^{(0)}=P^{(0)} w^{(0)}$ and the symmetry conditions, we can consider the two parts of the forcing $P^{(0)} w^{(0)}$ separately and then linearly superimpose their solutions. The polynomial part of this forcing, $P^{(0)}\left(-\frac{1}{2} y^{2}+h y+h a_{0}^{(0)}\right)$, is satisfied by the quartic solution

$$
P^{(0)}\left[-\frac{1}{24}(y-h)^{4}+\frac{1}{2}\left(\frac{1}{2} h^{2}+h a_{0}^{(0)}\right)(y-h)^{2}\right],
$$

which is even about the channel centreline $y=h$, and $x=0,1$. In the infinite series part of the forcing, each term takes a form proportional to $\cosh \left[k_{n}(y-h)\right] \cos \left(k_{n} x\right)$, and so the particular solution in this case is

$$
f_{n}(x, y):=\frac{(y-h) \sinh \left[k_{n}(y-h)\right]}{2 k_{n}} \cos \left(k_{n} x\right)
$$

which is also even about $y=h$ and $x=0,1$. Note that each series term in the forcing is harmonic, so each corresponding particular solution $f_{n}$ is biharmonic $\left(\nabla^{4} f_{n}=0\right)$. Thus $f_{n}$ can also be found from the biharmonic general solution in Cartesian coordinates, and enforcing $\nabla^{2} f_{n}=\cosh \left(k_{n}(y-h)\right) \cos \left(k_{n} x\right)$ and symmetry. Finally, combining all these components we find

$$
\begin{aligned}
T_{p}^{(0)}= & P^{(0)}\left[-\frac{1}{24}(y-h)^{4}+\frac{1}{2}\left(\frac{1}{2} h^{2}+h a_{0}^{(0)}\right)(y-h)^{2}\right. \\
& \left.+\sum_{n=1}^{\infty} \frac{h a_{n}^{(0)}}{2 k_{n}} \frac{(y-h) \sinh \left(k_{n}(y-h)\right)}{\cosh \left(k_{n} h\right)} \cos \left(k_{n} x\right)\right],
\end{aligned}
$$

which satisfies (5.1). Recalling $T^{(0)}=T_{p}^{(0)}+\widetilde{T}^{(0)}$, the remaining part, $\widetilde{T}^{(0)}$, then satisfies the homogeneous equation

$$
\nabla^{2} \widetilde{T}^{(0)}=0, \quad 0<x<1, \quad 0<y<h,
$$


but with a modification to the condition on $y=0$, which can be written as $\partial_{y} \widetilde{T}^{(0)}(x, 0)=$ $-H(x-\delta)-\partial_{y} T_{p}^{(0)}(x, 0)$, or

$$
\partial_{y} \widetilde{T}^{(0)}(x, 0)=-H(x-\delta)+P^{(0)}\left\{Q^{(0)}+\sum_{n=1}^{\infty} \frac{h a_{n}^{(0)}}{2 k_{n}}\left[\tanh \left(k_{n} h\right)+k_{n} h\right] \cos \left(k_{n} x\right)\right\},
$$

where $H(x)$ is the Heaviside step function. Lastly, $\widetilde{T}^{(0)}$ satisfies the symmetry conditions (5.4).

Just as for the previous Laplace problems ((4.6) and (4.15)), $\widetilde{T}^{(0)}$ has a series solution,

$$
\widetilde{T}^{(0)}=b_{0}^{(0)}+\sum_{n=1}^{\infty} b_{n}^{(0)} \frac{\cosh \left[k_{n}(y-h)\right]}{\cosh \left(k_{n} h\right)} \cos \left(k_{n} x\right),
$$

and the coefficients $b_{n}^{(0)}$ can be determined from the condition (5.9) at $y=0$. The problems for $\tilde{w}^{(0)}$ and $\tilde{w}^{(1)}$ had mixed Dirichlet and Neumann conditions at $y=0$, and resulted in dual series equations. The condition (5.9) for $\widetilde{T}^{(0)}$ is not mixed, only discontinuous, and so can be handled in the usual way by substituting (5.10) into condition (5.9), then multiplying by $\cos \left(k_{m} x\right)$ and integrating over $x \in[0,1]$. The result is

$$
b_{m}^{(0)}=\frac{\operatorname{coth}\left(k_{m} h\right)}{k_{m}}\left\{-\frac{2 \sin \left(k_{m} \delta\right)}{k_{m}}-\frac{P^{(0)} h a_{m}^{(0)}}{2 k_{m}}\left[\tanh \left(k_{m} h\right)+k_{m} h\right]\right\}, \quad m=1,2, \ldots
$$

Note that since the boundary condition is in terms of $\partial_{y} \widetilde{T}^{(0)}$, it cannot determine the first coefficient, $b_{0}^{(0)}$. Instead, it is fixed by enforcing the zero mixed-mean condition, $T_{m}=0$, which to leading order is

$$
\int_{0}^{h} \int_{0}^{1} w^{(0)} T^{(0)} \mathrm{d} x \mathrm{~d} y=0 .
$$

This holds because we used the mixed-mean in the definition of $T$ as per (2.12). Substituting the series expressions for $w^{(0)}$ and $T^{(0)}$, and then using orthogonality and integration by parts gives

$$
\begin{aligned}
b_{0}^{(0)}= & -\frac{1}{Q^{(0)}}\left\{P^{(0)}\left[\frac{1}{336} h^{7}-\frac{7}{120}\left(\frac{1}{2} h^{2}+h a_{0}^{(0)}\right) h^{5}+\frac{1}{6}\left(\frac{1}{2} h^{2}+h a_{0}^{(0)}\right)^{2} h^{3}\right]\right. \\
& +\sum_{n=1}^{\infty} \frac{P^{(0)} h^{2}\left(a_{n}^{(0)}\right)^{2}}{4 k_{n}}\left[\frac{h}{4 k_{n}}\left(2-\operatorname{sech}^{2}\left(k_{n} h\right)\right)-\frac{1}{4 k_{n}^{2}} \tanh \left(k_{n} h\right)\right] \\
& \left.+\sum_{n=1}^{\infty} \frac{h a_{n}^{(0)} b_{n}^{(0)}}{2}\left[\frac{1}{2} h \operatorname{sech}^{2}\left(k_{n} h\right)+\frac{1}{2 k_{n}} \tanh \left(k_{n} h\right)\right]\right\}
\end{aligned}
$$

Therefore, given the Fourier coefficients $a_{n}^{(0)}$ of the velocity field $w^{(0)}$, the temperature field $T^{(0)}$ can be decomposed into a particular solution $T_{p}^{(0)}$, given explicitly by (5.7), and a Fourier series $\widetilde{T}^{(0)}$, with coefficients given explicitly by (5.11) and (5.13).

Setting $y=0$ in expressions (5.7) and (5.10) then gives the temperature $T_{s}^{(0)}(x)$ on 
the composite interface,

$$
\begin{aligned}
T_{s}^{(0)}(x)= & P^{(0)}\left[-\frac{1}{24} h^{4}+\frac{1}{2}\left(\frac{1}{2} h^{2}+h a_{0}^{(0)}\right) h^{2}+\sum_{n=1}^{\infty} \frac{h^{2} a_{n}^{(0)}}{2 k_{n}} \tanh \left(k_{n} h\right) \cos \left(k_{n} x\right)\right] \\
& +b_{0}^{(0)}+\sum_{n=1}^{\infty} b_{n}^{(0)} \cos \left(k_{n} x\right) .
\end{aligned}
$$

The local and average Nusselt numbers are given by $N u^{(0)}(x)=4 h / T_{s}^{(0)}(x)$ and $\overline{N u}^{(0)}=$ $\int_{\delta}^{1} 4 h / T_{s}^{(0)} \mathrm{d} x$, with the integration performed numerically.

\subsection{First-order solution, $T^{(1)}$}

In this section we develop the first-order correction, $T^{(1)}$, to the temperature field due to the curvature of the meniscus. From section 3, the problem for $T^{(1)}$ is:

$$
\begin{aligned}
\nabla^{2} T^{(1)} & =P^{(1)} w^{(0)}+P^{(0)} w^{(1)}, & & 0<x<1, \quad 0<y<h, \\
\partial_{y} T^{(1)}(x, 0) & =\eta \partial_{y y} T^{(0)}(x, 0)-\eta^{\prime} \partial_{x} T^{(0)}(x, 0), & & 0 \leqslant x<\delta, \\
\partial_{y} T^{(1)}(x, 0) & =0, & & \delta<x \leqslant 1,
\end{aligned}
$$

along with vanishing normal derivatives on the remaining boundaries,

$$
\partial_{x} T^{(1)}(0, y)=\partial_{x} T^{(1)}(1, y)=\partial_{y} T^{(1)}(x, h)=0 .
$$

$P^{(0)}$ and $P^{(1)}$ are constants given by $(3.22)$, in terms of the flow rates $Q^{(0)}, Q_{1}^{(1)}$, and $Q_{2}^{(1)}$ of the velocity problem. Note that the boundary condition (5.16) for $T^{(1)}$ on the meniscus comes from expanding the normal derivative condition $\boldsymbol{n} \cdot \boldsymbol{\nabla} T=0$, and so takes the same form as that for $w^{(1)},(3.9)$. The condition (5.16) can be simplified in a similar fashion to (3.9) by noting from the series solution that $\nabla^{2} T^{(0)}=P^{(0)} w^{(0)}$ applies not only in the domain interior but also on $y=0,0 \leqslant x<\delta$ (excluding the triple contact line, i.e., $y=0, x=\delta)$. Thus (5.16) can be written

$$
\partial_{y} T^{(1)}(x, 0)=-\partial_{x}\left[\eta \partial_{x} T^{(0)}(x, 0)\right]+\eta P^{(0)} w^{(0)}(x, 0), \quad 0 \leqslant x<\delta .
$$

To proceed, we take the same approach that we took for $T^{(0)}$, and set $T^{(1)}=T_{p}^{(1)}+\widetilde{T}^{(1)}$ where $T_{p}^{(1)}$ is the particular solution that satisfies the forcing in $(5.15)$, and $\widetilde{T}^{(1)}$ is a remaining homogeneous (harmonic) part that enforces the conditions at $y=0$. In (5.15), the first forcing term, $P^{(1)} w^{(0)}$, is the same as at the previous order, (5.1), with $P^{(0)}$ replaced with $P^{(1)}$. The second term, $P^{(0)} w^{(1)}$, is similar, but with $w^{(0)}$ replaced with $w^{(1)}$, which has the same Fourier series structure. Thus we can construct $T_{p}^{(1)}$ from two copies of $T_{p}^{(0)}$, with the above changes made. It can be verified that

$$
\begin{aligned}
T_{p}^{(1)}= & P^{(1)}\left[-\frac{1}{24}(y-h)^{4}+\frac{1}{4} h^{2}(y-h)^{2}\right]+\frac{1}{2} h\left(P^{(1)} a_{0}^{(0)}+P^{(0)} a_{0}^{(1)}\right)(y-h)^{2} \\
& +\sum_{n=1}^{\infty} \frac{h}{2 k_{n}}\left(P^{(1)} a_{n}^{(0)}+P^{(0)} a_{n}^{(1)}\right) \frac{(y-h) \sinh \left[k_{n}(y-h)\right]}{\cosh \left(k_{n} h\right)} \cos \left(k_{n} x\right),
\end{aligned}
$$

satisfies $\nabla^{2} T_{p}^{(1)}=P^{(1)} w^{(0)}+P^{(0)} w^{(1)}$, and the symmetry conditions (5.18). The remaining part, $\widetilde{T}^{(1)}$, in the decomposition then satisfies the homogeneous problem

$$
\nabla^{2} \widetilde{T}^{(1)}=0, \quad 0<x<1, \quad 0<y<h,
$$


but with the following modification to the conditions on $y=0$,

$$
\partial_{y} \widetilde{T}^{(1)}(x, 0)=H(x-\delta)\left[\eta P^{(0)} w^{(0)}(x, 0)-\partial_{x}\left(\eta \partial_{x} T^{(0)}(x, 0)\right)\right]-\partial_{y} T_{p}^{(1)}(x, 0),
$$

where the last term is from differentiating (5.20). Similarly to the previous Laplace problems $\left(\tilde{w}^{(0)}, \tilde{w}^{(1)}\right.$, and $\left.\widetilde{T}^{(0)}\right), \widetilde{T}^{(1)}$ has a series solution

$$
\widetilde{T}^{(1)}=b_{0}^{(1)}+\sum_{n=1}^{\infty} b_{n}^{(1)} \frac{\cosh \left[k_{n}(y-h)\right]}{\cosh \left(k_{n} h\right)} \cos \left(k_{n} x\right),
$$

and the $b_{n}^{(1)}$ are found from condition (5.22) by subtituting (5.20), then multiplying by $\cos \left(k_{m} x\right)$ (for $m \geqslant 1$ ) and integrating $x \in[0,1]$ giving

$$
\begin{aligned}
b_{m}^{(1)}= & \operatorname{coth}\left(k_{m} h\right)\left[\frac{4 h}{k_{m}} P^{(0)} a_{0}^{(0)}\left(\frac{\delta \cos \left(k_{m} \delta\right)}{k_{m}^{2}}-\frac{\sin \left(k_{m} \delta\right)}{k_{m}^{3}}\right)-\sum_{n \geqslant 1} \frac{2 h}{k_{m}} P^{(0)} a_{n}^{(0)} \tilde{L}_{m n}\right. \\
& \left.-\sum_{n \geqslant 1}\left(h^{2} P^{(0)} a_{n}^{(0)} \tanh \left(k_{n} h\right)+2 k_{n} b_{n}^{(0)}\right) L_{m n}\right] \\
& -\frac{h}{2 k_{m}^{2}}\left(P^{(1)} a_{m}^{(0)}+P^{(0)} a_{m}^{(1)}\right)\left[1+k_{m} h \operatorname{coth}\left(k_{m} h\right)\right], \quad m=1,2, \ldots
\end{aligned}
$$

where $L_{m n}=\int_{0}^{\delta} \eta(x) \cos \left(k_{m} x\right) \cos \left(k_{n} x\right) \mathrm{d} x$ and $\tilde{L}_{m n}=\int_{0}^{\delta} \eta(x) \sin \left(k_{m} x\right) \sin \left(k_{n} x\right) \mathrm{d} x$ are known matrices, given by (A 1) and (A 2), respectively (see appendix A). As with $\widetilde{T}^{(0)}$, the coefficient $b_{0}^{(1)}$ is fixed by the zero mixed-mean condition. Expanding this condition for $0<\epsilon \ll 1$ :

$$
\begin{aligned}
0= & \int_{A} w(x, y ; \epsilon) T(x, y ; \epsilon) \mathrm{d} x \mathrm{~d} y \\
= & \int_{0}^{h} \int_{0}^{1} w T \mathrm{~d} x \mathrm{~d} y+\int_{0}^{\delta} \int_{-\epsilon \eta}^{0} w T \mathrm{~d} y \mathrm{~d} x \\
= & \int_{0}^{h} \int_{0}^{1} w^{(0)} T^{(0)} \mathrm{d} x \mathrm{~d} y+\epsilon \int_{0}^{h} \int_{0}^{1}\left(w^{(0)} T^{(1)}+w^{(1)} T^{(0)}\right) \mathrm{d} x \mathrm{~d} y \\
& +\epsilon \int_{0}^{\delta} \eta w^{(0)}(x, 0) T^{(0)}(x, 0) \mathrm{d} x+O\left(\epsilon^{2}\right) .
\end{aligned}
$$

At $O\left(\epsilon^{0}\right)$ this recovers (5.12), and at $O\left(\epsilon^{1}\right)$ we have

$$
0=\int_{0}^{h} \int_{0}^{1}\left(w^{(0)} T^{(1)}+w^{(1)} T^{(0)}\right) \mathrm{d} x \mathrm{~d} y+\int_{0}^{\delta} \eta w^{(0)}(x, 0) T^{(0)}(x, 0) \mathrm{d} x .
$$

Substituting our series solutions for $w^{(0)}, w^{(1)}, T^{(0)}$, and $T^{(1)}$ gives an expression for $b_{0}^{(1)}$. The computation is straightforward but long, and the expression for $b_{0}^{(1)}$ is unwieldy, so the details can be found in appendix B. Despite this, the expression (B 1) for $b_{0}^{(1)}$ is explicit and given exactly in terms of the velocity coefficients, $a_{n}^{(0)}$ and $a_{n}^{(1)}$, and the geometric parameters $h$ and $\phi($ or $\delta=1-\phi)$.

Defining $T_{s}^{(1)}(x):=T^{(1)}(x, 0)$, the corrections to the local and average Nusselt numbers are found numerically from $N u^{(1)}=-4 h T_{s}^{(1)} /\left(T_{s}^{(0)}\right)^{2}$ and $\overline{N u}^{(1)}=$ $-\int_{\delta}^{1} 4 h T_{s}^{(1)} /\left(T_{s}^{(0)}\right)^{2} \mathrm{~d} x$, respectively. 


\section{Asymptotic solution for $h \rightarrow \infty$}

In addition to the solution for arbitrary channel height $h$, a simple yet accurate asymptotic solution in the limit $h \rightarrow \infty$ can be found. With the goal of avoiding numerically solving the dual series equations, which converge very slowly (see figure 13), we seek explicit asymptotic solutions for $\lambda=\lambda^{(0)}+\epsilon\left(\lambda_{1}^{(1)}+\lambda_{2}^{(1)}\right)+\cdots$ and $\overline{N u}=\overline{N u}^{(0)}+\epsilon \overline{N u}^{(1)}+\cdots$, now assuming the channel height is large. As $h \rightarrow \infty$, an asymptotic expansion of each quantity in powers of $h^{-1}$ would capture all algebraic dependence on $h$, albeit neglecting exponentially small terms. The resulting limits of $w^{(0)}, w^{(1)}, T^{(0)}, T^{(1)}$ will depend on whether $y=O(1)$ or $y=O(h)$ as $h \rightarrow \infty$. If $y=O(1), y$ is fixed on the scale of the ridge period. If $y=O(h)$, then $y$ is fixed on the scale of (half) the channel height, and so remains in the central bulk of the channel as $h \rightarrow \infty$. In the language of matched asymptotic expansions, the former is the "inner region" and the latter is the "outer region." The following can be derived formally by constructing asymptotic solutions in each region and matching, or by taking $h \rightarrow \infty$ in the solutions already presented and neglecting exponentially small terms. For brevity we will present the latter approach. A remarkable finding of this analysis is that a two term asymptotic expansion for the slip length is accurate with exponentially small errors. As a result we produce formulas that are shown to be accurate for $h$ as small as 0.5, making them useful for engineering applications.

\subsection{Zeroth-order velocity}

First consider the dual series equations (4.10)-(4.11) for $a_{n}^{(0)}$. The part of (4.11) preventing an analytical solution is the $\tanh (n \pi h)$ factor appearing in the sum. But $\tanh (n \pi h) \sim 1+$ e.s.t. as $h \rightarrow \infty$, where "e.s.t." denotes "exponentially small terms." So replacing $\tanh (n \pi h)$ with 1 , the equations are those for a semi-infinite channel (coefficients denoted $a_{n}^{(0, \infty)}$ ) solved by Sbragaglia \& Prosperetti (2007). Thus $a_{n}^{(0)} \sim$ $a_{n}^{(0, \infty)}+$ e.s.t.. For $y=O(1)$ (the inner region), taking $h \rightarrow \infty$ in the solution (4.5) for $w^{(0)}$ :

where

$$
w^{(0)} \sim h\left(y+\tilde{w}^{(0, \infty)}\right)-\frac{1}{2} y^{2}+\text { e.s.t., } \quad \text { as } h \rightarrow \infty
$$

$$
\tilde{w}^{(0, \infty)}(x, y)=a_{0}^{(0, \infty)}+\sum_{n=1}^{\infty} a_{n}^{(0, \infty)} e^{-n \pi y} \cos (n \pi x) .
$$

We note that $\tilde{w}^{(0, \infty)}$ is the perturbation to the shear-driven flow in a semi-infinite domain, with slip length $\lambda^{(0, \infty)}=a_{0}^{(0, \infty)}$, given by Philip (1972):

$$
\tilde{w}^{(0, \infty)}(x, y)=\operatorname{Im}\left\{\frac{2}{\pi} \cos ^{-1}\left[\frac{\cos \left(\frac{\pi z}{2}\right)}{\cos \left(\frac{\pi \delta}{2}\right)}\right]\right\}, \quad \text { where } \quad z=x+i y .
$$

In particular,

$$
\begin{aligned}
& a_{0}^{(0, \infty)}=\frac{2}{\pi} \log \left[\sec \left(\frac{\pi \delta}{2}\right)\right], \\
& \tilde{w}^{(0, \infty)}(x, 0)=\frac{2}{\pi} \cosh ^{-1}\left[\frac{\cos \left(\frac{\pi x}{2}\right)}{\cos \left(\frac{\pi \delta}{2}\right)}\right], \quad 0 \leqslant x<\delta, \\
& {\frac{\partial \tilde{w}^{(0, \infty)}}{\partial x}}^{(x, 0)=-\frac{\sqrt{2} \sin \left(\frac{\pi x}{2}\right)}{\sqrt{\cos \pi x-\cos \pi \delta}},} \quad 0 \leqslant x<\delta .
\end{aligned}
$$


For $y=O(h)$ (the outer region), write $y=h Y$ with $Y=O(1)$ (in fact $Y \leqslant 1$ due to the geometry of the problem). In this region, the sum in $w^{(0)}$ is exponentially small, so

$$
w^{(0)} \sim-\frac{1}{2} h^{2} Y^{2}+h^{2} Y+h a_{0}^{(0, \infty)}+\text { e.s.t., } \quad h \rightarrow \infty
$$

which is the Navier slip velocity profile.

\subsection{First-order velocity}

Using $\tanh (n \pi h) \sim 1+$ e.s.t. and $\tilde{w}^{(0)}(x, 0) \sim \tilde{w}^{(0, \infty)}(x, 0)+$ e.s.t. in the dual series equations (4.20)-(4.21) for $a_{n}^{(1)}$, we obtain

$$
\begin{aligned}
a_{0}^{(1)}+\sum_{n=1}^{\infty} a_{n}^{(1)} \cos (n \pi x)=0, & \delta<x<1 \\
\sum_{n=1}^{\infty} a_{n}^{(1)} n \pi \cos (n \pi x)=\partial_{x}\left(\eta \partial_{x} \tilde{w}^{(0, \infty)}(x, 0)\right)+\frac{\eta}{h}+\text { e.s.t., } & 0<x<\delta .
\end{aligned}
$$

These can now also be solved analytically, in the manner of Sbragaglia \& Prosperetti (2007), to give

$$
\begin{gathered}
\lambda_{1}^{(1)}=a_{0}^{(1)} \sim \underbrace{-\delta^{3} \int_{0}^{1} \frac{[1-\cos (\delta \pi s)]\left(1-s^{2}\right) \mathrm{d} s}{\cos (\delta \pi s)-\cos (\delta \pi)}}_{a_{0}^{(1, \infty)}}+\frac{1}{h} \sqrt{2} \delta^{4} \int_{0}^{1} \frac{s\left(1-s^{2} / 3\right) \sin (\delta \pi s / 2) \mathrm{d} s}{\sqrt{\cos (\delta \pi s)-\cos (\delta \pi)}} \\
+ \text { e.s.t., } \quad h \rightarrow \infty .
\end{gathered}
$$

Sbragaglia \& Prosperetti only obtained the first term, $a_{0}^{(1, \infty)}$, producing an $O\left(h^{-1}\right)$ error. But if the $O\left(h^{-1}\right)$ term is captured, as above, the error is exponentially small - a remarkable improvement. The solution of (6.8)-(6.9) can then be used in asymptotic expressions for $w^{(1)}$ in the inner and outer regions, just as for $w^{(0)}$.

Lastly, an exponentially accurate expression for $\lambda_{2}^{(1)}$, the slip due to the increase in cross-sectional area, is given by

$$
\begin{aligned}
\lambda_{2}^{(1)} & \sim \frac{1}{h} \int_{0}^{\delta} \tilde{w}^{(0, \infty)}(x, 0) \eta(x) \mathrm{d} x+\text { e.s.t. } \\
& =\frac{1}{h} \sqrt{2} \delta^{4} \int_{0}^{1} \frac{s\left(1-s^{2} / 3\right) \sin (\delta \pi s / 2) \mathrm{d} s}{\sqrt{\cos (\delta \pi s)-\cos (\delta \pi)}}+\text { e.s.t. }
\end{aligned}
$$

which is $O\left(h^{-1}\right)$ and, remarkably, identical to the second term in the expression (6.10) for $\lambda_{1}^{(1)}$. Thus $\lambda^{(1)}=\lambda_{1}^{(1)}+\lambda_{2}^{(1)} \sim a_{0}^{(1, \infty)}+2 \lambda_{2}^{(1)}$.

\subsection{Nusselt numbers}

Neglecting only exponentially small terms, inner and outer asymptotic solutions as $h \rightarrow \infty$ can be found for the temperature field in a similar manner to the velocity field, but the results are not as useful. For exponential accuracy, or even to go further than $O\left(h^{-1}\right)$ in the expansion, however, the full solution to the dual series equations (6.8)-(6.9) is needed, which we only know numerically (analytically we only know $a_{0}^{(1)}$ in the limit $h \rightarrow \infty)$. Using the asymptotic behaviours $a_{0}^{(0)} \sim \lambda^{(0, \infty)}+$ e.s.t, $a_{n}^{(0)} \sim a_{n}^{(0, \infty)}+$ e.s.t, $P^{(0)} \sim \phi /\left(h^{3} / 3+h^{2} \lambda^{(0, \infty)}\right)+$ e.s.t. from the velocity problem in the series expression 
(5.14) for $T_{s}^{(0)}(x)$, we find (after dividing by $h$ )

$$
\frac{1}{h} T_{s}^{(0)}(x)=\frac{17}{35} \phi-\frac{18}{35} \frac{\phi}{h} \lambda^{(0, \infty)}+\frac{1}{h} \sum_{n=1}^{\infty} b_{n}^{(0, \infty)} \cos (n \pi x)+O\left(\frac{1}{h^{2}}\right) .
$$

Here $b_{n}^{(0, \infty)}$ is the leading order of $b_{n}^{(0)}$ as $h \rightarrow \infty$, and $b_{n}^{(0)} \sim b_{n}^{(0, \infty)}+O\left(h^{-1}\right) \sim$ $-2 \sin (n \pi \delta) /(n \pi)^{2}+O\left(h^{-1}\right)$. Subsituting into (3.25), expanding further for $h \rightarrow \infty$ and integrating, we find correct to $O\left(h^{-1}\right)$,

$$
\overline{N u}^{(0)} \sim \frac{140}{17}\left[1+\frac{1}{h} \frac{35}{17}\left(\frac{18}{35} \lambda^{(0, \infty)}-\sum_{n=1}^{\infty} \frac{2 \sin ^{2}(n \pi \delta)}{\phi^{2}(n \pi)^{3}}\right)\right]+O\left(\frac{1}{h^{2}}\right),
$$

Also, using the behaviour of $a_{0}^{(1)}, a_{n}^{(1)}$, and $b_{n}^{(1)}$ as $h \rightarrow \infty$ in the series expression for $T_{s}^{(1)}(x)$, and then substituting into (3.25), we find after some algebra,

$$
\overline{N u}^{(1)} \sim\left(\frac{35}{17}\right)^{2} \frac{4}{h}\left[\frac{18}{35} a_{0}^{(1, \infty)}+\sum_{n=1}^{\infty} \frac{b_{n}^{(1, \infty)} \sin ^{2}(n \pi \delta)}{\phi^{2} n \pi}\right]+O\left(\frac{1}{h^{2}}\right),
$$

where

$$
b_{n}^{(1, \infty)}=4 \sum_{n=1}^{\infty} L_{n m} \frac{\sin (m \pi \delta)}{m \pi},
$$

$\lambda^{(0, \infty)}=a_{0}^{(0, \infty)}$ is $(6.4), a_{0}^{(1, \infty)}$ is in (6.10), and $L_{n m}$ is given by (A 1$)$. Despite only $O\left(h^{-1}\right)$ accuracy, these expressions are explicit, with only the sums requiring evaluation.

\section{Results and discussion}

In this section we present results for the effect of meniscus curvature on the slip length and Nusselt number, representative of the velocity and temperature problems, respectively. At both orders in $\epsilon$, we quantify the dependence on slip fraction $\delta$ (or equivalently solid fraction $\phi=1-\delta$ ) and channel height $h$, and assess the accuracy of the asymptotic solutions for $h \rightarrow \infty$.

\subsection{Apparent slip length}

In the literature, results for the slip length are usually presented normalised by the period, so we will present the quantity $\lambda^{*} / 2 d^{*}=\lambda / 2$. Figure 4 shows plots of the slip lengths $\lambda^{(0)}, \lambda_{1}^{(1)}, \lambda_{2}^{(1)}$ (normalised by ridge period) against the channel height $h$, for various slip fractions $\delta$. Also shown, as dashed lines, are the asymptotic solutions for $h \rightarrow \infty$, given by (6.4),(6.10) and (6.11). The numerical and asymptotic solutions agree extremely well: less than $1 \%$ difference is typically achieved by $h=1$ for $\lambda^{(0)}$, and already by $h=0.5$ for $\lambda_{1}^{(1)}$ and $\lambda_{2}^{(1)}$. This attests to the exponential accuracy of the asymptotic solutions. The asymptotics should be exact in the limit $h \rightarrow \infty$, and the slight difference seen to persist in the curves for $\lambda_{1}^{(1)}$ is numerical error of the series solution due to the slow convergence with $N$, the number of terms used. Hence, even for $N=7000$, the number of terms used in figure 4 , the asymptotic solution may actually be more accurate than the numerical solution for $h \gg 1$.

The behaviour of $\lambda^{(0)}$ is well known (see Teo \& Khoo 2009; Maynes \& Crockett 2014), and $\lambda_{1}^{(1)}$ and $\lambda_{2}^{(1)}$ behave similarly to the case of ridges on only one wall (see Sbragaglia $\&$ Prosperetti 2007). Recall that $\lambda_{1}^{(1)}$ represents the effect of meniscus curvature on the 

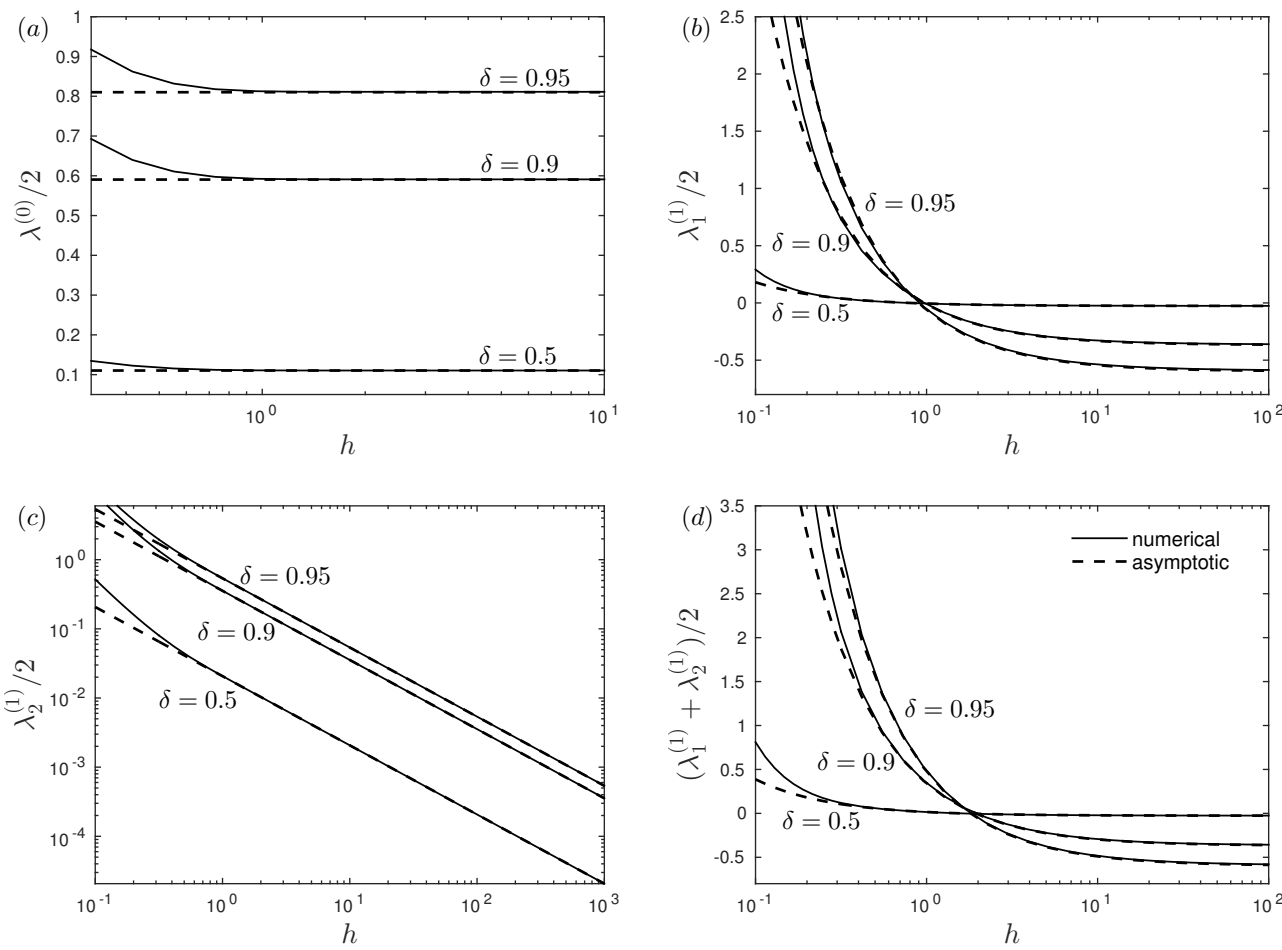

FiguRE 4. Slip lengths (normalised by period) against the channel height $h$ for increasing values of slip fraction $\delta=1-\phi$. Panels $(a)-(c)$ correspond to quantities (4.27)-(4.29), respectively. Solid lines are numerical solutions of the dual series equations, dashed lines are asymptotic solutions (6.4), (6.10), (6.11) for $h \gg 1$. Panel $(d)$ shows the full first-order perturbation. Calculations used $N=7000$.

velocity field. The asymptotic behaviour (6.10) of $\lambda_{1}^{(1)}$ is of the form $\lambda_{1}^{(1)} \sim-A(\delta)+$ $B(\delta) h^{-1}$, where $A$ and $B$ are positive (increasing) functions of $\delta$. It is positive for $h \lesssim 1$, and negative for $h \gtrsim 1$, tending to a constant negative value, $\lambda_{1}^{(1, \infty)}=-A$ as $h \rightarrow \infty$. An estimate of when $\lambda_{1}^{(1)}$ changes sign is given by $h \approx B / A$. Physically this means that the effect on the velocity field of the meniscus protuding slightly into $(\epsilon>0)$ the gas cavity is to reduce slip for thicker channels $(h \gtrsim 1)$, and increase slip for narrower channels $(h \lesssim 1)$. If the meniscus protrudes out of the cavity $(\epsilon<0)$, then the reverse is true.

On the other hand, the quantity $\lambda_{2}^{(1)}$, representing the change in flow rate due to the change in cross-sectional area, has asymptotic behaviour $\lambda_{2}^{(1)} \sim B(\delta) h^{-1}$ (see $(6.11)$ ), which is positive, and vanishes in the limit $h \rightarrow \infty$. Thus, for a meniscus protruding into the cavity, the increase in cross-sectional area always increases slip, but the effect vanishes in the large channel limit.

Finally, the full first-order perturbation with $\lambda_{1}^{(1)}$ and $\lambda_{2}^{(1)}$ combined, $\lambda^{(1)}=\lambda_{1}^{(1)}+$ $\lambda_{2}^{(1)} \sim-A(\delta)+2 B(\delta) h^{-1}$, is shown in figure $4(d)$, with similar behaviour to $\lambda_{1}^{(1)}$, but changing sign at $h \approx 2 B / A$.

For an illustration of how the velocity field changes due to curvature, contour plots of the velocity correction $\tilde{w}^{(1)}$ throughout the domain cross-section, and along the meniscus, are shown in figure 5 . For visualisations of $\tilde{w}^{(0)}$ see Teo \& Khoo (2009). Note that $w^{(1)}(x, 0)=h \tilde{w}^{(1)}(x, 0)$ is the order $\epsilon$ correction to the velocity on the meniscus, since 

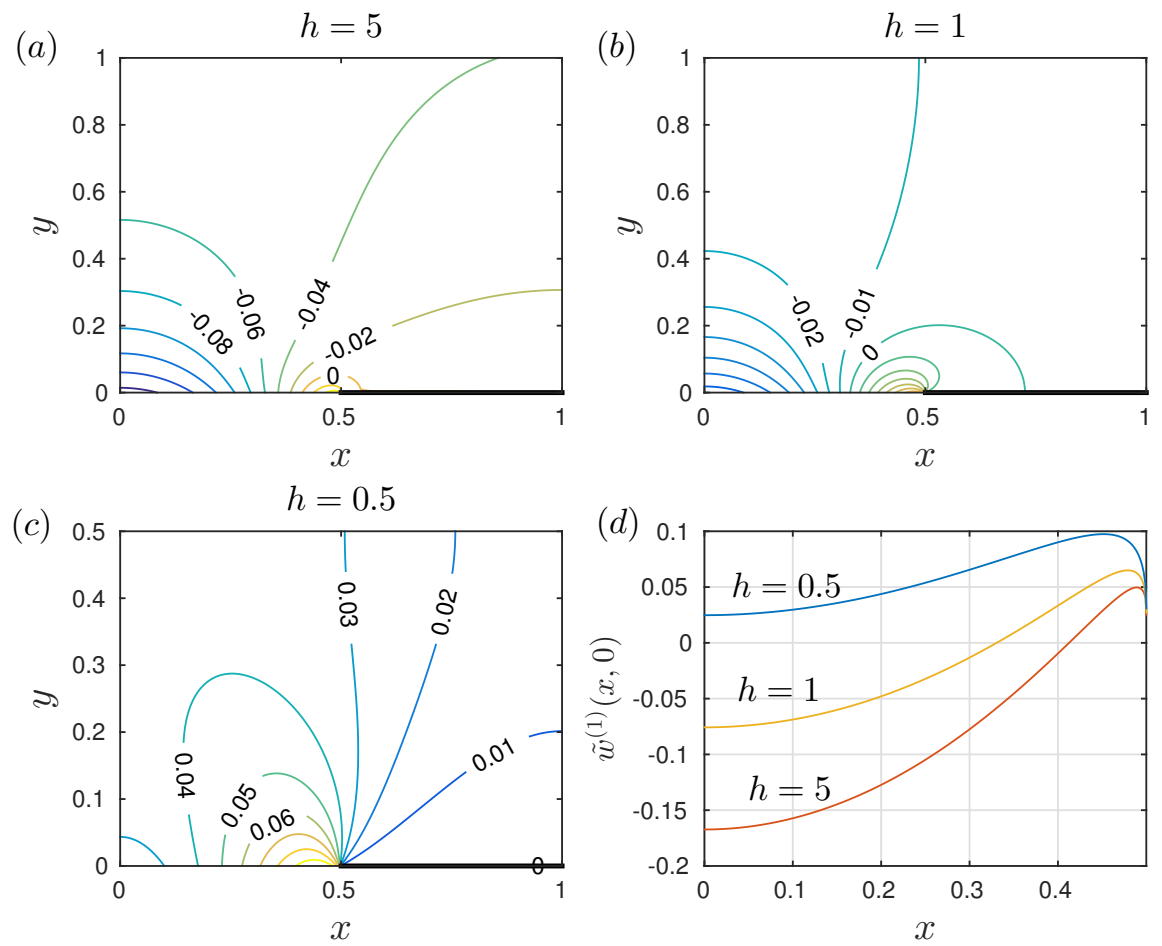

FIGURE 5. First-order velocity field $\tilde{w}^{(1)}(x, y)$ for a solid fraction $\phi=0.5$, and channel heights $h=5,1,0.5$ in panels $(a)-(c)$. Note the $y$ scales. Black solid line marks the position of the ridge. Panel $(d)$ shows the velocity along the meniscus, $0 \leqslant x \leqslant 0.5, y=0$. Here $N=7000$.

$\partial_{y} w^{(0)}(x, 0)=0$. As figure 5 shows, the flow is always increased in the vicinity of the triple contact line $\left(\tilde{w}^{(1)}>0\right)$, but flow can be either increased $\left(\tilde{w}^{(1)}>0\right)$ or decreased $\left(\tilde{w}^{(1)}<0\right)$ throughout the rest of the domain, depending on $h$. Indeed, $\tilde{w}^{(1)}$ increases everywhere in the domain as $h$ decreases, giving rise to the behaviour of $\lambda_{1}^{(1)}$ in figure $4(b)$, where it eventually becomes positive.

\subsection{Leading order Nusselt number behaviour}

The recent study by Maynes \& Crockett (2014) considered the (isoflux) thermal problem for the case of a flat meniscus, corresponding to the $T^{(0)}$ problem in our boundary perturbation formulation, but they used the one-dimensional Navier slip velocity profile rather than the exact two-dimensional velocity profile, $w^{(0)}$. The Navier slip profile corresponding to $w^{(0)}$, i.e., with the same flow rate, is given by $w_{N S}^{(0)}=-\frac{1}{2} y^{2}+h y+h \lambda^{(0)}$. It is identical to $w^{(0)}$ absent the infinite sum, which contributes nothing to the flow rate $Q^{(0)}$. Therefore, the approximate solution of Maynes \& Crockett corresponds exactly to replacing $\left(a_{0}^{(0)}, a_{1}^{(0)}, a_{2}^{(0)}, \ldots\right)$ with $\left(a_{0}^{(0)}, 0,0, \ldots\right)$ in the expressions above. In addition, to save having to compute $a_{0}^{(0)}=\lambda^{(0)}$ numerically, the value for a semi-infinite $(h \rightarrow \infty)$ channel, $\lambda^{(0, \infty)}=(2 / \pi) \log [\sec (\delta \pi / 2)]$, was used.

The justification by Maynes \& Crockett for using the Navier slip profile is that the velocity field becomes independent of $x$ very quickly as $y$ increases from zero, becoming indistinguishable from the Navier slip profile above a distance of approximately one ridge period (Teo \& Khoo 2009). When the channel height is comparable to the wall period, 

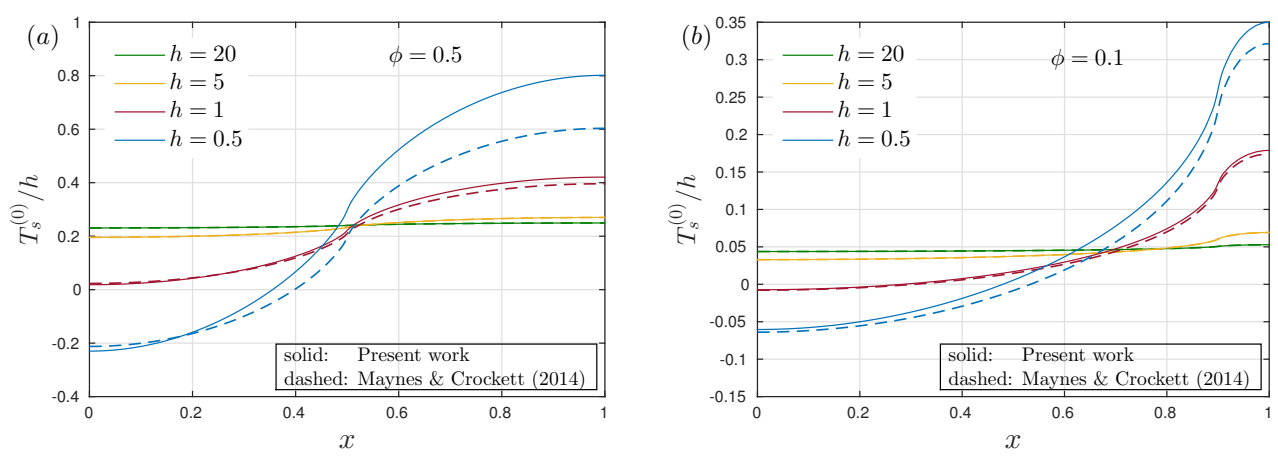

Figure 6. (Colour online) The leading order temperature, $T_{s}^{(0)}(x) / h$, along the flat composite interface, $y=0$, for channel heights $h=20,5,1,0.5$. The solid lines are results calculated using the full velocity field, and the dashed lines are calculated using the Navier slip profile with slip length $\lambda^{(0, \infty)}=(2 / \pi) \log [\sec (\pi \delta / 2)]$, as in Maynes \& Crockett $(2014)$. Number of terms is $N=120$. Panels $(a)$ and $(b)$ are for solid fractions $\phi=0.5$ and 0.1 , respectively.

$h=O(1)$, they argued that heat diffusion away from the ridges $\left(\widetilde{T}^{(0)}\right)$ dominates over advection $\left(T_{p}^{(0)}\right)$, and so the exact form of the velocity profile is irrelevant. This was checked by trying other less accurate profiles, such as the no-slip profile, which produced $\mathrm{a} \leqslant 0.5 \%$ difference in $\overline{N u}^{(0)}$. However, the $x$ variation of the velocity field $w^{(0)}$ does not just enter the wall temperature through the advective part $\left(T_{p}^{(0)}\right)$ but also through $\widetilde{T}^{(0)}$ - see (5.11) and (5.13). Thus having $T_{p}^{(0)}$ small relative to $\widetilde{T}^{(0)}$ does not in and of itself imply the effect of $x$ variation is negligible.

For domains with height much larger than the wall period $(h \gg 1)$, Maynes \& Crockett argued that the advection terms dominate, in which case the Navier slip profile prevails throughout the vast majority of the channel, and thus is a good approximation. Checking against numerical solutions for each case showed a maximum error in $\overline{N u}^{(0)}$ of $1.6 \%$. However, the numerical solutions did not account for $x$ variation of the velocity, and so no conclusion regarding $x$ variation can be made, despite the small errors stated. The check must be done against the full solution using the full two-dimensional profile, and this is carried out next.

The solution presented here accounts for the full velocity profile, and thus is valid for arbitrary channel heights $h$, including $h=O(1)$. We extend the results of Maynes \& Crockett (2014) down to $h=O(1)$, and show for what parameter values the Navier slip approximation is sufficient. We could consider the case of $h \ll 1$, but in practice there is a lower limit to $h$ such that the surface structure stays dewetted, i.e., in the Cassie state (Cottin-Bizonne et al. 2004). For $h \rightarrow \infty$ it can be seen from (5.7) and (5.10) that $T^{(0)}=O(h)$, and so to connect with the relevant finite temperature field in the limit of large channels we consider $T^{(0)} / h$. Also, this quantity is more relevant to the Nusselt number, with the local Nusselt number over the solid ridge given by $N u^{(0)}(x)=4 h / T_{s}^{(0)}(x)$ (and zero over the meniscus).

The temperature, $T_{s}^{(0)}(x) / h$, along the flat composite interface, $y=0$, is shown in figure 6 , for various channel heights, $h$, from $h=20$ down to $h=0.5$. Figure $6(a)$ is for a solid fraction $\phi=0.5$, and figure $6(b)$ is for a lower value of $\phi=0.1$. Results using the full velocity field, and those using the Navier slip approximation with slip length $\lambda^{(0, \infty)}=(2 / \pi) \log [\sec (\pi \delta / 2)]$ as in Maynes \& Crockett $(2014)$, are both shown. The temperature appears approximately constant across the composite interface for $h>5$, 

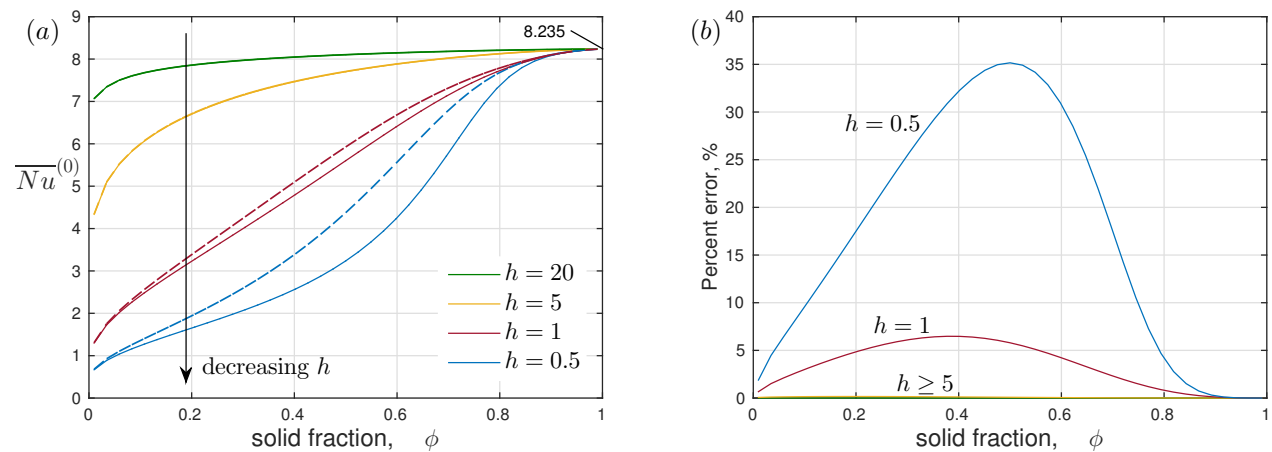

Figure 7. (Colour online) The leading order average Nusselt number, $\overline{N u}^{(0)}$, against solid fraction $\phi$ for various $h$. See caption for figure 6. Panel $(a)$ compares the present work (solid lines) to the approach of Maynes \& Crockett (2014) (dashed lines), and (b) shows the relative percentage error.

with the channel height showing little influence. As $h$ decreases, the temperature increases over the ridge, and decreases over the meniscus. Using the Navier slip solution appears to be a good approximation for $h \gtrsim 5$, with the temperatures indistinguishable, but the discrepancy with the full solution is visible for smaller $h$, becoming larger as $h$ decreases. The Navier slip approximation underestimates the temperature over the ridge and most of the cavity, which could be because the slip profile overestimates the velocity just above the ridge (where the flow in reality satisfies no-slip) where the heat is entering the domain. Hence the exact velocity field would convect heat downstream and away from its point of entrance more slowly, raising the temperature immediately above the ridge (and part-way over the cavity by conduction). In addition, the Navier slip profile underestimates the velocity over the meniscus, but as no heat is entering through the meniscus (assumed adiabatic), this has less of an effect.

The effect of neglecting the $x$ variation in the velocity on the average Nusselt number, $\overline{N u}^{(0)}$, is shown in figure $7(a)$. The relative percent error is shown in figure $7(b)$. Note that only the temperature over the solid $(1-\phi<x<1)$ contributes to the calculation of $\overline{N u}^{(0)}$. In general, for any channel height, $\overline{N u}^{(0)}$ decreases as the solid fraction decreases, its maximum value of $140 / 17 \approx 8.235$ attained when there is no meniscus - the classical uniform heating result (Kays et al. 2005). $\overline{N u}^{(0)}$ stays close to this value for large $h$ (asymptotics show $\overline{N u}^{(0)} \sim 140 / 17+O\left(h^{-1}\right)$ as $h \rightarrow \infty$ ), but decreases as $h$ decreases, signifying an overall increase in thermal resistance as the channel is made narrower. The Navier slip approximation is valid for sufficiently large $h$, with an error in $\overline{N u}^{(0)}$ of $\leqslant 0.16 \%$ for $h \geqslant 5$. But the discrepancy increases as $h$ decreases, with up to $6.48 \%$ overestimation when $h=1$, and up to $35.2 \%$ overestimation when $h=0.5$ (when channel height matches period). The error is greatest for intermediate solid fractions $\phi$, but disappears as $\phi \rightarrow 0$ (no ridge) and $\phi \rightarrow 1$ (no meniscus). This is because the Navier slip velocity coincides with the actual velocity in both limits. For $h=0.5$, when the error is significant, figure 8 illustrates the wall temperature for various $\phi$, with the greatest discrepancy for intermediate values of $\phi$.

In conclusion, the results of Maynes \& Crockett (2014) are accurate for large channel heights $(h \geqslant 5)$ with very small error, but overestimate $\overline{N u}^{(0)}$ and hence the overall convective heat transfer by up to $35 \%$ when $h=O(1)$. This means, for flat menisci, the superhydrophobic structuring reduces convective heat transfer more than previously 


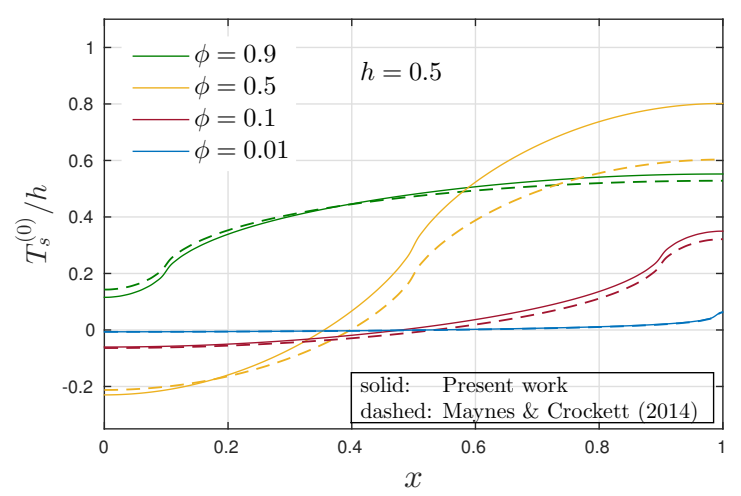

Figure 8. (Colour online) The leading order temperature, $T_{s}^{(0)} / h$, along the flat composite interface, $y=0$, for various values of solid fraction $\phi$, at channel height $h=0.5$. Solid/dashed carry the same meaning as in figures 7,6 .

documented compared to classical uniform heating. However, the results are relatively unchanged for very small solid fractions $\phi$, where lubrication is highest, since the error is $\leqslant 2.5 \%$ for $\phi<0.01$.

\subsection{First-order Nusselt number behaviour}

We now present the results for the first-order temperature problem, and hence the effect of small meniscus curvature on the average Nusselt number, $\overline{N u}$. First we show the correction to the temperature (scaled by $h$ ) along the composite interface, given by $T_{s}^{(1)}(x) / h$. We remark that even though $T_{s}^{(0)}(x)$ and $T_{s}^{(1)}(x)$ are evaluated at $y=0$, the combination $T_{s}^{(0)}(x)+\epsilon T_{s}^{(1)}(x)$ corresponds to the temperature on the curved interface $y=-\epsilon \eta(x)$ when $0<x<1-\phi$, correct to order $\epsilon$. Also, recall that $\epsilon>0$ (or negative protrusion angle $\theta$ ) corresponds to the meniscus protruding into the cavity, and $\epsilon<0$ (or positive protrusion angle $\theta$ ) corresponds to the meniscus protruding into the liquid. Hence, if $\epsilon>0$, then $T_{s}^{(1)}>0$ implies an increase in temperature compared to a flat meniscus, and $T_{s}^{(1)}<0$ implies a decrease. (The opposite if $\epsilon<0$.)

Figure 9 shows $T_{s}^{(1)} / h$, for the same $h$ and $\phi$ values as in figure 6 . For any $h, T_{s}^{(1)}>0$ over the centre of the meniscus, and, for most parameter values shown, $T_{s}^{(1)}<0$ over the entirety of the solid ridge. However, $T_{s}^{(1)}$ is most negative on the edges of the meniscus, just before the triple contact line. This means, if the meniscus curves into the cavity $(\epsilon>$ 0 ), the temperature is increased over the centre of the meniscus, but decreased at its edges and over the solid. The temperature increase over the centre of the meniscus may surprise the reader, given its distance from the ridge, but the increase is just the consequence of the decrease near the ridge and enforcing zero mixed-mean dimensionless temperature as per (2.12). Indeed, the same qualitative behaviour is seen for pure heat diffusion in the absence of advection, but we do not include the results here. As $h$ is reduced and the channel gets thinner, these effects are enhanced. But for $h$ small enough $(h \approx 0.5$ in $9 a), T_{s}^{(1)}$ can become positive over the solid, and thus increase the temperature. This is surprising, and the physical cause is unclear.

The first-order correction, $\overline{N u}^{(1)}$, to the average Nusselt number is plotted in figure 10 against solid fraction, $\phi$, for the same values of $h$ as in figure 7 . In general, decreasing $\phi$ increases $\overline{N u}^{(1)}$, meaning greater enhancement to convection for wider cavities. For 

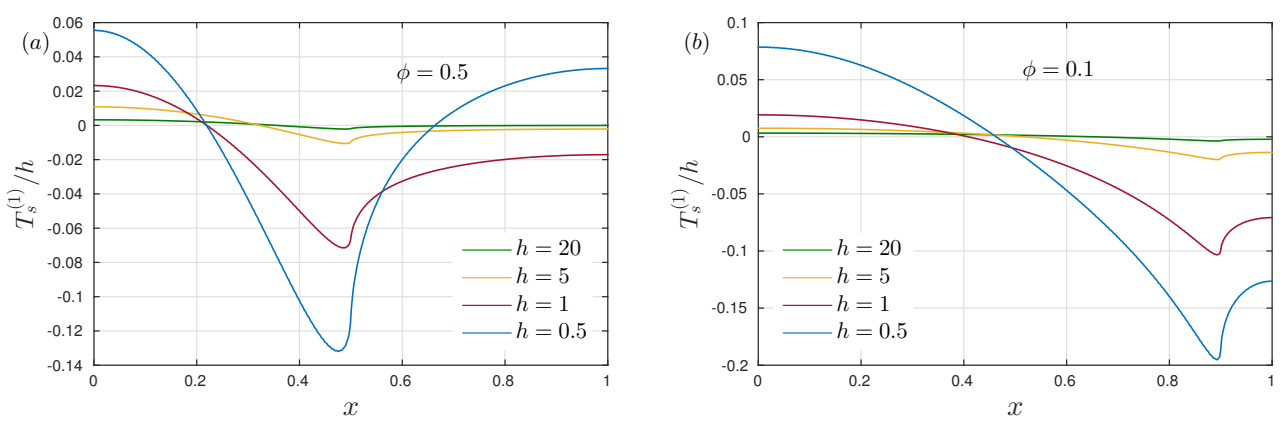

Figure 9. (Colour online) The first-order temperature correction, $T_{s}^{(1)} / h$, along the composite interface for values of channel height $h=20,5,1,0.5$. Panel $(a)$ and $(b)$ are for solid fractions $\phi=0.5$ and 0.1 , respectively. In all cases, number of terms used is $N=500$.

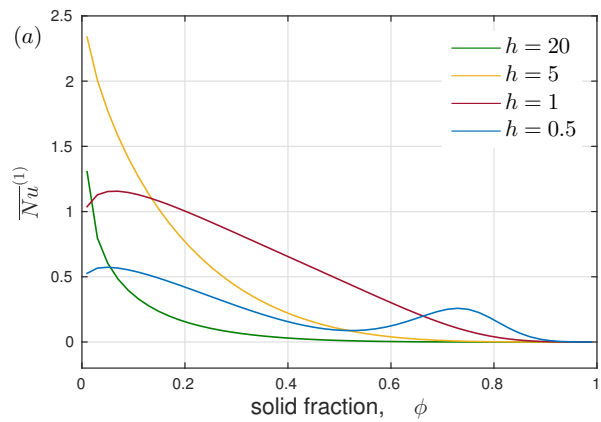

Figure 10. (Colour online) The first-order average Nusselt number correction, $\overline{N u}^{(1)}$, against solid fraction $\phi$ for various $h$. Number of terms $N=500$.

$\phi>0.5$, and $h$ small enough, we can see a local maximum emerge, but in the limit $\phi \rightarrow 1$, we see $\overline{N u}^{(1)} \rightarrow 0$, as expected since the cavity and meniscus disappear.

The effect of channel height, $h$, is more easily discerned in figure 11 where $\overline{N u}^{(1)}$ is plotted with $h$ as the dependent variable - panel $(a)$ shows the very low $h$ behaviour (included for completeness), and panel $(b)$ the range $h \geqslant 0.5$ (more physically relevant). In figure $11(b)$, the solid lines are $\overline{N u}^{(1)}$, and the dashed lines are $\overline{N u}^{(0)}$, which are included for reference. As $h \rightarrow \infty$, we have $\overline{N u}^{(1)}=O\left(h^{-1}\right) \rightarrow 0$, which can be seen from the asymptotic solution, (6.14). Then as $h$ decreases from $\infty, \overline{N u}^{(1)}$ increases to a maximum, typically between $h=1$ and $h=10$, then falls again as $h$ decreases further. In other words, $\overline{N u}^{(1)}$ behaves non-monotonically with respect to $h$, with the curvature presenting the greatest enhancement for $1 \lesssim h \lesssim 5$.

To understand this non-monotonicity, consider the two regimes $h \gg 1, h \ll 1$. When the channel height is large but finite, $h \gg 1$, the heat transfer is approximately diffusive on the scale of the ridges, with advection only significant in the main body of the channel, where the velocity is approximately one dimensional (a Navier slip profile). In this regime, a downward protruding meniscus affects convection in two competing ways: (i) by decreasing the lubrication as seen by the Navier slip profile, and (ii) increasing the cross-sectional area into which heat can diffuse, near the triple contact line in particular. These effects appear as the two $O\left(h^{-1}\right)$ terms in the expression (6.14) for $\overline{N u}^{(1)}$ as $h \rightarrow \infty$. Comparison of the two terms shows that effect (ii) is always greater than effect 

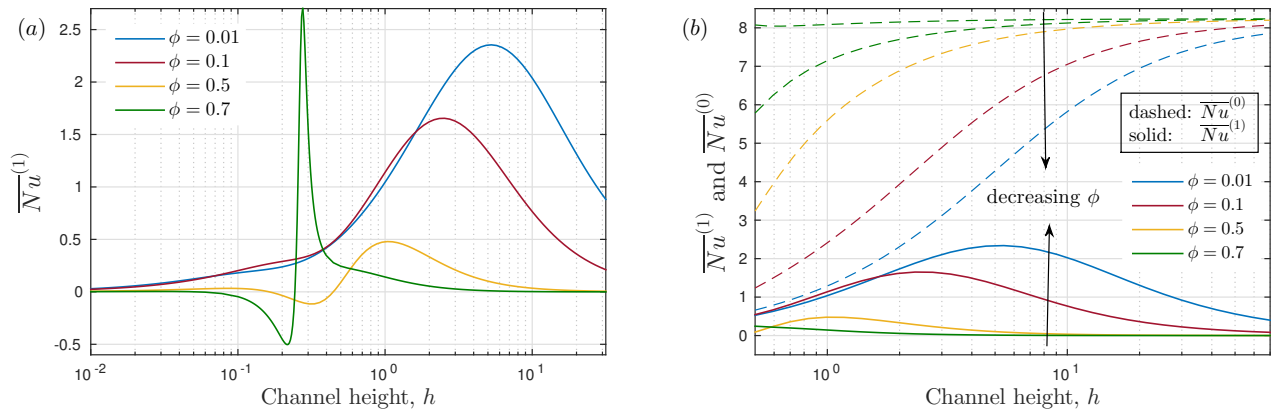

Figure 11. (Colour online) The first-order average Nusselt number correction, $\overline{N u}^{(1)}$ (solid lines), against channel height $h$ for various $\phi$. Panel (a) shows small $h$ behaviour, and $(b)$ shows $h \geqslant 0.5$. Dashed lines are $\overline{N u}^{(0)}$. Here $N=500$.

(i), and so convection is enhanced, $\overline{N u}^{(1)}>0$. Both effects vanish for a semi-infinite channel, $h \rightarrow \infty$, and so $\overline{N u}^{(1)} \rightarrow 0^{+}$. For the case when the channel height is small but finite, $h \ll 1$, the ridges on opposing walls are almost touching. In the region between opposing ridges, the fluid velocity decreases as $h$ decreases (no-slip is imposed on both ridges). However, flow in the region between opposing menisci does not degrade as $h$ decreases (for example, $w^{(0)} \rightarrow\left(\delta^{2}-x^{2}\right) / 2$ as $h \rightarrow 0$ ). Therefore, the heat must diffuse from the ridges through the stagnant region between opposing ridges into the region between menisci. Menisci protruding into the cavities $(\epsilon>0)$ would then increase the flow rate between menisci, and the area for heat diffusion from the ridge-ridge region, enhancing convection, $\overline{N u}^{(1)}>0$. In the limit $h \rightarrow 0$, the ridges touch, the region between the ridges vanishes and the heat has no way of reaching the region of flow, and so $\overline{N u}^{(1)} \rightarrow 0^{+}$, regardless of meniscus curvature.

For the two above physical limits, i.e., $\overline{N u}^{(1)} \rightarrow 0^{+}$as $h \rightarrow 0$ and $h \rightarrow \infty$, to be achieved, $\overline{N u}^{(1)}$ is necessarily non-monotonic in $h$. Bridging the aforementioned regimes is the case of $h=O(1)$, where there is a nontrivial interaction between advection, meniscus curvature, and channel geometry, as presented in figure 11(b).

Indeed, we also found that for a sufficiently high solid fraction (see figure 11a), $\overline{\mathrm{Nu}}^{(1)}$ becomes negative for an interval of $h$ less than 0.45 . The values of solid fraction $\phi$ and channel height $h$ for which this occurs (within the region $\phi>0.402$ and $h<0.45$ of parameter space) are not practical for applications, and we have not been able to explain it physically, but this behaviour is mathematically interesting. Given the two asymptotic behaviours $\overline{N u}^{(1)} \rightarrow 0^{+}$(i.e., from above) as $h \rightarrow 0$ and $h \rightarrow \infty$, the unusual sign changes possible in $\overline{N u}^{(1)}$ would have been difficult to anticipate, and display the complex nonlinear dependence of the problem on both $h$ and $\phi$.

Comparison between the numerical solutions for $\overline{N u}^{(0)}$ and $\overline{N u}^{(1)}$ and asymptotic expressions (6.13) and (6.14) as $h \rightarrow \infty$ are shown in figure 12. Although not as accurate as our asymptotic slip length solutions, since the error is estimated to be $O\left(h^{-2}\right)$, the $O\left(h^{-1}\right)$ behaviour for $\overline{N u}^{(0)}$ and $\overline{N u}^{(1)}$ is confirmed. The asymptotic approximation for $\overline{N u}^{(0)}$ does much better than that for $\overline{N u}^{(1)}$, but the approximation for $\overline{N u}^{(1)}$ improves for smaller solid fractions, which is surprising.

The aforementioned results show that meniscus curvature can enhance/reduce the overall heat transfer, but the role of changes in advection alone are difficult to assess. One 

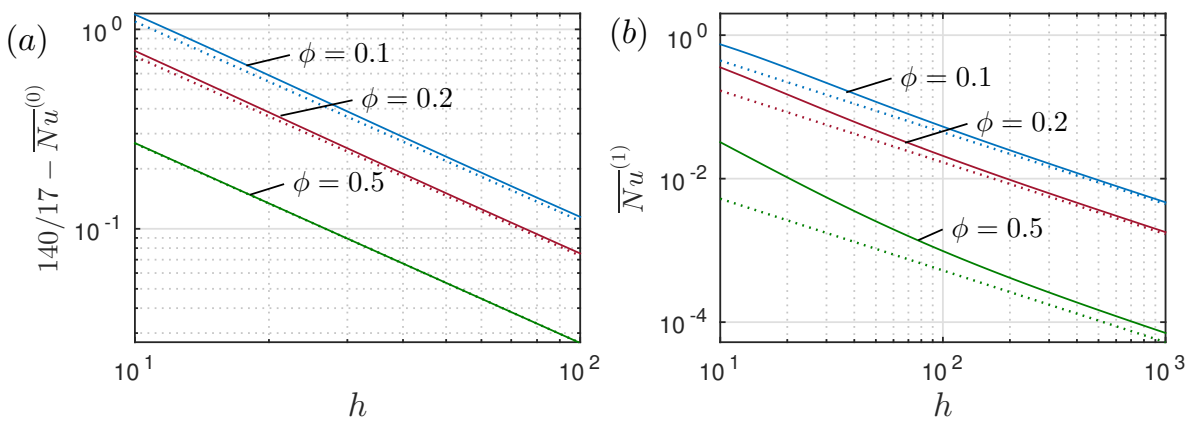

Figure 12. (Colour online) Comparison between numerical solution (solid lines) for $\overline{N u}^{(0)}, \overline{N u}^{(1)}$ and asymptotic solution (dotted lines) as $h \rightarrow \infty$, given by (6.13),(6.14).

would expect the behaviour to be apparent in the correction to the velocity field, $\tilde{w}^{(1)}$. But as figure 5 shows, the flow is always increased in the vicinity of the triple contact line $\left(\tilde{w}^{(1)}>0\right)$, but flow can be either increased $\left(\tilde{w}^{(1)}>0\right)$ or decreased $\left(\tilde{w}^{(1)}<0\right)$ throughout the rest of the domain, depending on $h$. As $h$ is decreased, $\tilde{w}^{(1)}$ becomes more positive, first over the ridge (figure $5 b$ ), then eventually the whole cross-section (figure $5 c$ ). This could explain the initial increase in $\overline{N u}^{(1)}$, but not its subsequent decrease for $h \lesssim 1$, as $\tilde{w}^{(1)}$ continues to increase everywhere. Indeed, $T^{(1)}$ and $\overline{N u}^{(1)}$ depend not only on the velocity change, $\tilde{w}^{(1)}$, but the base velocity and temperature, $\tilde{w}^{(0)}$ and $T^{(0)}$, all of which can interact to produce inscrutable sign changes as shown in figure 11.

\subsection{Remark on convergence of series}

The solutions developed in this paper are based on truncating infinite series at $n=N$, where $N$ is chosen large enough for the solutions to have sufficiently converged. The rate of convergence will thus depend on the decay of the terms in the series. The terms in each series solution considered here all decay exponentially with $n$ if $y>0$, i.e., in the interior of the domain. This decay becomes algebraic when $y=0$, i.e., on the composite interface. For example, $a_{n}^{(0)}$ are the Fourier coefficients of $w^{(0)}(x, 0)$, which can be shown to have a square root singularity in its derivative at the triple contact line, i.e., $w^{(0)}(x, 0)=O\left(|x-\delta|^{1 / 2}\right)$, as $x \rightarrow \delta^{-}$(Philip 1972). This implies $a_{n}^{(0)}=O\left(n^{-\frac{3}{2}}\right)$ as $n \rightarrow \infty$. The decay in the series for $w^{(1)}(x, 0)$ is governed by $a_{n}^{(1)}$, and the behaviour of $w^{(1)}(x, 0)$ as $x \rightarrow \delta^{-}$can be determined from a local analysis of the full solution, $w$, close to the singularity. Close to the singularity $x=\delta$, the domain locally takes the form of Laplace's equation in a wedge with interior angle $\pi-\theta$ (see figure 1 ). The problem can be easily solved by conformally mapping to the first quadrant, from which we find, on the meniscus, $w=O\left(r^{\pi / 2(\pi-\theta)}\right)$ as $r \rightarrow 0$, where $r$ is the distance to the singularity. An expansion for small angle $\theta$, should then yield the local behaviour of $w^{(0)}$ and $w^{(1)}$ :

$$
\begin{aligned}
r^{\frac{\pi}{2(\pi-\theta)}} & =r^{1 / 2}+\frac{\theta}{2 \pi} r^{1 / 2} \log r+O\left(\theta^{2}\right), \\
& =r^{1 / 2}-\frac{\epsilon \delta}{\pi} r^{1 / 2} \log r+O\left(\epsilon^{2}\right) .
\end{aligned}
$$

This suggests $w^{(1)}=O\left(r^{1 / 2} \log r\right)$, and that its Fourier coefficients behave as $a_{n}^{(1)}=$ $O\left(n^{-3 / 2} \log n\right)$ as $n \rightarrow \infty$, which is confirmed in figure 13(a). Note that the expansion in $\theta$ above breaks down for $r$ sufficiently small, i.e., $r \sim e^{-\frac{1}{\theta}}$, when the terms become 

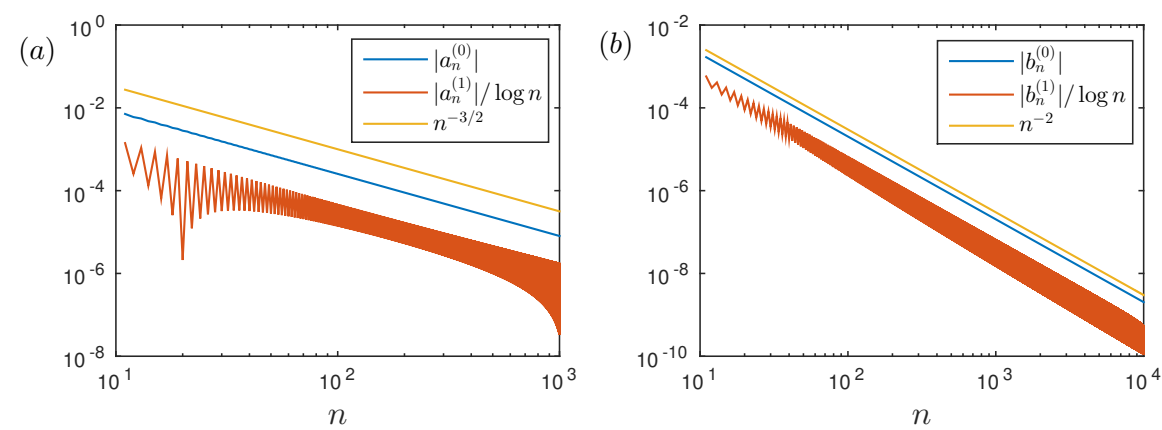

Figure 13. (Colour online) Decay rates as $n \rightarrow \infty$ for the coefficients $a_{n}^{(0)}, a_{n}^{(1)}, b_{n}^{(0)}, b_{n}^{(1)}$ for $N=10^{4}, h=10, \phi=0.5$. Velocity coefficients shown in $(a)$, temperature coefficients in $(b)$.

disordered. This suggests that a small angle expansion does not capture the behaviour exponentially close to the singularity.

Using these dependencies for $a_{n}^{(0)}$ and $a_{n}^{(1)}$, the expressions in section 5.1 give: $b_{n}^{(0)}=$ $O\left(n^{-2}\right)$ for the series terms in $\widetilde{T}^{(0)}$, and $O\left(n^{-\frac{5}{2}}\right)$ for those in $T_{p}^{(0)}$. Hence the $b_{n}^{(0)}$ limits the convergence of $T^{(0)}$. At the next order, our numerics suggest (see figure 13) that $b_{n}^{(1)}=O\left(n^{-2} \log n\right)$. Similarly to $w$, the behaviour of $T$ near $x=\delta$, and hence $T^{(0)}$ and $T^{(1)}$, can also be found via a local analysis.

All this implies the first-order series converge slower than the leading order series, by a factor of $\log n$, and the velocity series converge slower than the temperature series, by a factor of $n^{1 / 2}$. This is reflected in the results where, to achieve graphical accuracy with a laptop computer, we needed $N=7000$ (with several minutes of computation time) for the first-order slip lengths, but only $N=120$ and $N=500$ (with less than a second per plot) for $\overline{N u}^{(0)}$ and $\overline{N u}^{(1)}$.

\section{Conclusions}

The effect of small meniscus curvature on the thermal transport in microchannels with longitudinal grooved surfaces has been studied. Employing a boundary perturbation method, the full velocity and temperature fields and their first-order corrections were found in the form of eigenfunction expansions, reducing to dual series equations for the expansion coefficients. The desired quantities representing the influence of the ridge structuring for the hydrodynamic and thermal problems are the slip length $\lambda$, and Nusselt number $N u$, respectively.

For the hydrodynamic problem, the hydrodynamic slip length for a flat meniscus $\lambda^{(0)}$, and the first-order corrections due to meniscus curvature, $\lambda_{1}^{(1)}$ and $\lambda_{2}^{(1)}$, were presented as functions of channel height $h$, and solid fraction $\phi$, the parameters describing the geometry of the channel. They were computed both by numerically solving for the expansion coefficients, but also asymptotically for large channel heights, $h \gg 1$. Whereas the numerical slip length solutions show slow convergence to the true solution (up to $O\left(10^{4}\right)$ terms needed even for graphical accuracy), the asymptotic solutions (6.10)-(6.11) are only two terms, explicit, easily calculated, and remarkably accurate. Even though they were derived for $h \rightarrow \infty$, the error is exponentially small, so their validity can be extended down to $h \approx 0.5$, with very small error. This makes them useful for engineering applications. In agreement with previous work for longitudinal ridges (Sbragaglia \& Prosperetti 2007; Teo \& Khoo 2010), meniscus protrusion into the cavities decreases 
the overall slip length for large channel heights, but can increase the slip length for sufficiently narrow channels $(h \lesssim 2)$. This is not only due to a change in the velocity field itself, but also the increase in cross-sectional area becoming important.

In the thermal problem, eigenfunction expansions for the temperature field were derived in terms of the velocity solution, and thus accounted for advection. The resulting average Nusselt number for a flat meniscus, $\overline{N u}^{(0)}$, and first-order correction due to mensicus curvature, $\overline{N u}^{(1)}$, were then presented for a range of channel heights $h$, and solid fractions $\phi$. The aformentioned results are completely captured in a single graph, figure 11(b). At leading order (the case of a flat meniscus), we extend the results of Maynes \& Crockett (2014), who used an approximate velocity profile. We find that their approximation is accurate for large channel heights $(h \gtrsim 5)$ with very small error, but it overestimates $\overline{N u}^{(0)}$ and hence the overall convective heat transfer by up to $35 \%$ when $h=O(1)$. This means, for flat menisci, the superhydrophobic structuring reduces convective heat transfer more than previously documented compared to classical uniform heating. However, the results are relatively unchanged for very small solid fractions $\phi$, where lubrication is highest, since the error is $<2.5 \%$ for $\phi<0.01$. (see figure 7 ).

The general effect of meniscus curvature on the heat transfer problem agrees with that seen in the recent study by Lam et al. (2016), which used the same boundary perturbation approach for the thermal problem but in a semi-infinite domain $(h=\infty)$ without advection. When the meniscus protrudes into (out of) the cavity, there is an enhancement (reduction) in heat transfer, which is most prominent for small solid fractions. The results presented here for arbitrary $h$ show that the thermal transport is further enhanced (reduced) when the channel height is decreased down the scale of the ridges. The behaviour is non-monotonic in $h$, with the greatest enhancement (reduction) occuring for $1 \lesssim h \lesssim 10$, i.e., heights between 1 and 10 times the ridge period.

T.L.K. was supported by an EPSRC doctoral scholarship; M.H. was supported in part by NSF grant no. 1402783, and D.T.P. was supported in part by EPSRC grants $\mathrm{EP} / \mathrm{L} 020564 / 1$ and EP/K041134/1.

\section{Appendix A. Matrices $L_{m n}$ and $\tilde{L}_{m n}$}

Of relevance to the $b_{n}^{(1)}$ coefficients, $(5.24)$, the arrays $L_{m n}$ and $\tilde{L}_{m n}$ are (recall $k_{n}=$ $n \pi):$

$$
\begin{aligned}
L_{m n}= & \int_{0}^{\delta} \eta(x) \sin \left(k_{m} x\right) \sin \left(k_{n} x\right) \mathrm{d} x \\
= & \begin{cases}-\frac{\delta \cos \left(k_{m}-k_{n}\right) \delta}{\left(k_{m}-k_{n}\right)^{2}}+\frac{\sin \left(k_{m}-k_{n}\right) \delta}{\left(k_{m}-k_{n}\right)^{3}}+\frac{\delta \cos \left(k_{m}+k_{n}\right) \delta}{\left(k_{m}+k_{n}\right)^{2}} & m \neq n, \\
-\frac{\sin \left(k_{m}+k_{n}\right) \delta}{\left(k_{m}+k_{n}\right)^{3}}, & m=n . \\
\frac{1}{3} \delta^{3}+\frac{\delta \cos \left(k_{m}+k_{n}\right) \delta}{\left(k_{m}+k_{n}\right)^{2}}-\frac{\sin \left(k_{m}+k_{n}\right) \delta}{\left(k_{m}+k_{n}\right)^{3}}, & m\end{cases}
\end{aligned}
$$




$$
\begin{aligned}
\tilde{L}_{m n}= & \int_{0}^{\delta} \eta(x) \cos \left(k_{m} x\right) \cos \left(k_{n} x\right) \mathrm{d} x \\
= & \begin{cases}-\frac{\delta \cos \left(k_{m}-k_{n}\right) \delta}{\left(k_{m}-k_{n}\right)^{2}}+\frac{\sin \left(k_{m}-k_{n}\right) \delta}{\left(k_{m}-k_{n}\right)^{3}}-\frac{\delta \cos \left(k_{m}+k_{n}\right) \delta}{\left(k_{m}+k_{n}\right)^{2}} & m \neq n, \\
+\frac{\sin \left(k_{m}+k_{n}\right) \delta}{\left(k_{m}+k_{n}\right)^{3}}, & m=n . \\
\frac{1}{3} \delta^{3}-\frac{\delta \cos \left(k_{m}+k_{n}\right) \delta}{\left(k_{m}+k_{n}\right)^{2}}+\frac{\sin \left(k_{m}+k_{n}\right) \delta}{\left(k_{m}+k_{n}\right)^{3}}, & m\end{cases}
\end{aligned}
$$

\section{Appendix B. Calculation of coefficient $b_{0}^{(1)}$}

In this appendix we detail the calculation of the first coefficient, $b_{0}^{(1)}$, in the eigenfunction expansion for $\widetilde{T}^{(1)}$. It is determined by the zero mixed-mean temperature condition at $O\left(\epsilon^{1}\right)$, given by $(5.25)$, which we write as $I_{1}+I_{2}+I_{3}=0$, where

$$
\begin{aligned}
& I_{1}=\int_{0}^{h} \int_{0}^{1} w^{(0)} T^{(1)} \mathrm{d} x \mathrm{~d} y, \\
& I_{2}=\int_{0}^{h} \int_{0}^{1} w^{(1)} T^{(0)} \mathrm{d} x \mathrm{~d} y, \\
& I_{3}=\int_{0}^{\delta} \eta(x) w^{(0)}(x, 0) T^{(0)}(x, 0) \mathrm{d} x .
\end{aligned}
$$

As $T^{(1)}$ is identical in structure to $T^{(0)}$, the calculation of $I_{1}$ is similar to the leading order calculation, (5.12), giving

$$
\begin{aligned}
I_{1}= & P^{(1)}\left(\frac{13}{840} h^{7}+\frac{3}{80} a_{0}^{(0)} h^{6}\right)+\frac{1}{2} h\left(P^{(1)} a_{0}^{(0)}+P^{(0)} a_{0}^{(1)}\right)\left[\frac{1}{15} h^{5}+\frac{1}{3} h^{4} a_{0}^{(0)}\right] \\
& +\sum_{n \geqslant 1} \frac{h^{2}}{4 k_{n}} a_{n}^{(0)}[P a]_{n}\left[\frac{h}{4 k_{n}}\left(2-\operatorname{sech}^{2}\left(k_{n} h\right)\right)-\frac{1}{4 k_{n}^{2}} \tanh \left(k_{n} h\right)\right] \\
& +\sum_{n \geqslant 1} \frac{1}{2} h a_{n}^{(0)} b_{n}^{(1)}\left(\frac{1}{2} h \operatorname{sech}^{2}\left(k_{n} h\right)+\frac{1}{2 k_{n}} \tanh \left(k_{n} h\right)\right)+b_{0}^{(1)} Q^{(0)},
\end{aligned}
$$

where $[P a]_{n}:=P^{(1)} a_{n}^{(0)}+P^{(0)} a_{n}^{(1)}$. The first line is from the terms with no cosines; the first sum is from the product of the cosine series in $w^{(0)}$ with the cosine series in $T_{p}^{(1)}$; the second sum is from the product of the series in $w^{(0)}$ with the series in $\widetilde{T}^{(1)}$. The last term contains the only appearance of $b_{0}^{(1)}$. For $I_{2}$, since $w^{(1)}$ is very similar in structure to $w^{(0)}$, but missing the quadratic terms, the calculation is again similar, but even simpler:

$$
\begin{aligned}
I_{2}= & h P^{(0)} a_{0}^{(1)}\left[-\frac{1}{120} h^{5}+\frac{1}{6}\left(\frac{1}{2} h^{2}+h a_{0}^{(0)}\right) h^{3}\right] \\
& +\sum_{n \geqslant 1} \frac{h^{2}}{4 k_{n}} a_{n}^{(0)} P^{(0)} a_{n}^{(1)}\left[\frac{h}{4 k_{n}}\left(2-\operatorname{sech}^{2}\left(k_{n} h\right)\right)-\frac{1}{4 k_{n}^{2}} \tanh \left(k_{n} h\right)\right] \\
& +\sum_{n \geqslant 1} \frac{1}{2} h a_{n}^{(1)} b_{n}^{(0)}\left(\frac{1}{2} h \operatorname{sech}^{2}\left(k_{n} h\right)+\frac{1}{2 k_{n}} \tanh \left(k_{n} h\right)\right)+b_{0}^{(0)} Q_{1}^{(1)} .
\end{aligned}
$$


For $I_{3}$, the factor of $\eta(x)$ in the integrand prevents the use of the cosine orthogonality relations. Substituting $w^{(0)}$ and $T^{(0)}=T_{p}^{(0)}+\widetilde{T}^{(0)}$, then multiplying out and swapping the order of summation and integration, this becomes

$$
\begin{aligned}
I_{3}= & h a_{0}^{(0)}\left[P^{(0)}\left(\frac{5}{24} h^{4}+\frac{1}{2} a_{0}^{(0)} h^{3}\right)+b_{0}^{(0)}\right] \int_{0}^{\delta} \eta(x) \mathrm{d} x \\
& +\left[P^{(0)}\left(\frac{5}{24} h^{4}+\frac{1}{2} a_{0}^{(0)} h^{3}\right)+b_{0}^{(0)}\right] \sum_{n} h a_{n}^{(0)} \int_{0}^{\delta} \eta(x) \cos \left(k_{n} x\right) \mathrm{d} x \\
& +h a_{0}^{(0)} \sum_{n \geqslant 1}\left(\frac{h^{2}}{2 k_{n}} P^{(0)} a_{n}^{(0)} \tanh \left(k_{n} h\right)+b_{n}^{(0)}\right) \int_{0}^{\delta} \eta(x) \cos \left(k_{n} x\right) \mathrm{d} x \\
& +\sum_{n \geqslant 1} \sum_{m \geqslant 1} h a_{m}^{(0)}\left(\frac{h^{2}}{2 k_{n}} P^{(0)} a_{n}^{(0)} \tanh \left(k_{n} h\right)+b_{n}^{(0)}\right) \int_{0}^{\delta} \eta(x) \cos \left(k_{n} x\right) \cos \left(k_{m} x\right) \mathrm{d} x .
\end{aligned}
$$

Each integral can be calculated using integration by parts. To solve for the unknown, $b_{0}^{(1)}$, notice it only appears in the last term of $I_{1}$, and so $I_{1}+I_{2}+I_{3}=0$ is easily rearranged to give

$$
\begin{aligned}
-Q^{(0)} b_{0}^{(1)}= & P^{(1)}\left(\frac{13}{840} h^{7}+\frac{13}{120} a_{0}^{(0)} h^{6}+\frac{1}{6}\left(a_{0}^{(0)}\right)^{2} h^{5}\right)+P^{(0)} a_{0}^{(1)}\left(\frac{13}{120} h^{6}+\frac{1}{3} a_{0}^{(0)} h^{5}\right) \\
& +\frac{2}{3} \delta^{3} h a_{0}^{(0)}\left[P^{(0)}\left(\frac{5}{24} h^{4}+\frac{1}{2} a_{0}^{(0)} h^{3}\right)+b_{0}^{(0)}\right] \\
& +\sum_{n \geqslant 1}\left\{\frac{h^{2} a_{n}^{(0)}}{16 k_{n}^{3}}\left(2 P^{(0)} a_{n}^{(1)}+P^{(1)} a_{n}^{(0)}\right)\left[k_{n} h\left(2-\operatorname{sech}^{2}\left(k_{n} h\right)\right)-\tanh \left(k_{n} h\right)\right]\right. \\
& +\frac{1}{2} h\left(a_{n}^{(0)} b_{n}^{(1)}+a_{n}^{(1)} b_{n}^{(0)}\right)\left(\frac{1}{2} h \operatorname{sech}^{2}\left(k_{n} h\right)+\frac{1}{2 k_{n}} \tanh \left(k_{n} h\right)\right) \\
& +\left\{h a_{n}^{(0)}\left[P^{(0)}\left(\frac{5}{24} h^{4}+\frac{1}{2} a_{0}^{(0)} h^{3}\right)+b_{0}^{(0)}\right]\right. \\
& \left.\left.+h a_{0}^{(0)}\left[\frac{h^{2}}{2 k_{n}} P^{(0)} a_{n}^{(0)} \tanh \left(k_{n} h\right)+b_{n}^{(0)}\right]\right\}\left(-\frac{2 \delta}{k_{n}^{2}} \cos \left(k_{n} \delta\right)+\frac{2}{k_{n}^{3}} \sin \left(k_{n} \delta\right)\right)\right\} \\
& +\sum_{m \geqslant 1} \sum_{n \geqslant 1} h\left(\frac{h^{2}}{2 k_{n}} P^{(0)} a_{m}^{(0)} \tanh \left(k_{m} h\right)+b_{m}^{(0)}\right) \tilde{L}_{m n} a_{n}^{(0)} \\
& +Q_{1}^{(1)} b_{0}^{(0)} .
\end{aligned}
$$

\section{REFERENCES}

Baier, T., Steffes, C. \& Hardt, S. 2010 Thermocapillary flow on superhydrophobic surfaces. Phys. Rev. E 82 (3), 037301.

Bergman, T. L., Lavine, A. S., Incropera, F. P. \& DeWitt, D. P. 2011 Fundamentals of Heat and Mass Transfer, 7th edn. John Wiley \& Sons.

Cheng, Y., Xu, J. \& Sui, Y. 2015 Numerical study on drag reduction and heat transfer enhancement in microchannels with superhydrophobic surfaces for electronic cooling. App. Therm. Eng. 88 (SI), 71-81.

Cottin-Bizonne, C., Barentin, C., Charlaix, E., Bocquet, L. \& Barrat, J. L. 2004 Dynamics of simple liquids at heterogeneous surfaces: Molecular-dynamics simulations and hydrodynamic description. Eur. Phys. J. E 15 (4), 427-438. 
Crowdy, D. G. 2010 Slip length for longitudinal shear flow over a dilute periodic mattress of protruding bubbles. Phys. Fluids 22 (12), 121703.

Davies, J., Maynes, D., Webb, B. W. \& Woolford, B. 2006 Laminar flow in a microchannel with superhydrophobic walls exhibiting transverse ribs. Phys. Fluids $\mathbf{1 8}$ (8), 087110.

Davis, A. M. J. \& LAUGA, E. 2009 Geometric transition in friction for flow over a bubble mattress. Phys. Fluids 21 (1), 011701.

Enright, R., Eason, C., Dalton, T., Hodes, M., Salamon, T., Kolodner, P. \& KRUPENKIN, T. 2006 Friction factors and nusselt numbers in microchannels with superhydrophobic walls. In Proceedings of the 4 th International Conference on Nanchannels, Microchannels and Minichannels, Pts. A and B, pp. 599-609.

Enright, Ryan, Hodes, Marc, Salamon, Todd \& Muzychka, Yuri 2014 Isoflux nusselt number and slip length formulae for superhydrophobic microchannels. J. Heat Trans-T ASME 136 (1), 012402.

Hodes, M., Kirk, T., Karamanis, G., Lam, L., Maclachlan, S. \& Papageorgiou, D. $2015 a$ Conformal map and asymptotic solutions for apparent slip lengths in the presence of thermocapillary stress. In Proc. First International ISHMT-ASTFE Conference (IHMTC 2015), p. 1254.

Hodes, M., Lam, L. S., Cowley, A., Enright, R. \& MacLachlan, S. $2015 b$ Effect of evaporation and condensation at menisci on apparent thermal slip. J. Heat Trans-T ASME 137 (7), 071502.

Hodes, M., Zhang, R., Lam, L. S., Wilcoxon, R. \& Lower, N. 2014 On the potential of galinstan-based minichannel and minigap cooling. IEEE Trans. Compon. Packag. Technol. $4(1), 46-56$.

Kays, W., Crawford, M. \& Weigand, B. 2005 Convective Heat and Mass Transfer, 4th edn. McGraw-Hill, New York.

Lam, L. S., Hodes, M. \& Enright, R. 2015 Analysis of galinstan-based microgap cooling enhancement using structured surfaces. J. Heat Trans-T ASME 137 (9), 091003.

Lam, L. S., Hodes, M., Karamanis, G., Kirk, T. \& Maclachlan, S. 2016 Effect of meniscus curvature on apparent thermal slip. J. Heat Trans-T ASME p. under review.

Lauga, E. \& Stone, H. A. 2003 Effective slip in pressure-driven stokes flow. J. Fluid Mech. $489,55-77$.

Maynes, D. \& Crockett, J. 2014 Apparent temperature jump and thermal transport in channels with streamwise rib and cavity featured superhydrophobic walls at constant heat flux. J. Heat Trans-T ASME 136 (1), 011701.

Maynes, D., Jeffs, K., Woolford, B. \& WebB, B. W. 2007 Laminar flow in a microchannel with hydrophobic surface patterned microribs oriented parallel to the flow direction. Phys. Fluids 19 (9), 093603.

Maynes, D., Webb, B. W., Crockett, J. \& Solovjov, V. 2013 Analysis of laminar slip-flow thermal transport in microchannels with transverse rib and cavity structured superhydrophobic walls at constant heat flux. J. Heat Trans-T ASME 135 (2), 021701.

Maynes, D., WebB, B. W. \& Davies, J. 2008 Thermal transport in a microchannel exhibiting ultrahydrophobic microribs maintained at constant temperature. J. Heat Trans-T ASME $130(2), 022402$.

Ou, J., Perot, B. \& Rothstein, J. P. 2004 Laminar drag reduction in microchannels using ultrahydrophobic surfaces. Phys. Fluids 16 (12), 4635-4643.

Ou, J. \& Rothstein, J. P. 2005 Direct velocity measurements of the flow past drag-reducing ultrahydrophobic surfaces. Phys. Fluids 17 (10), 103606.

Philip, J. R. 1972 Flows satisfying mixed no-slip and no-shear conditions. Z. Angew. Math. Phys. 23 (3), 353-372.

Priezjev, N. V., Darhuber, A. A. \& Troian, S. M. 2005 Slip behavior in liquid films on surfaces of patterned wettability: Comparison between continuum and molecular dynamics simulations. Phys. Rev. E $\mathbf{7 1}$ (4), 41608.

Sbragaglia, M. \& Prosperetti, A. 2007 A note on the effective slip properties for microchannel flows with ultrahydrophobic surfaces. Phys. Fluids 19 (4), 043603.

Teo, C. J. \& Khoo, B. C. 2009 Analysis of stokes flow in microchannels with superhydrophobic surfaces containing a periodic array of micro-grooves. Microfluid. Nanofluidics 7 (3), 353382. 
Teo, C. J. \& Khoo, B. C. 2010 Flow past superhydrophobic surfaces containing longitudinal grooves: effects of interface curvature. Microfluid. Nanofluidics 9 (2-3), 499-511.

Tuckerman, D. B. \& Pease, R. F. W. 1981 High-performance heat sinking for VLSI. IEEE Electron Device Lett. 2 (5), 126-129.

Ybert, C., Barentin, C., Cottin-Bizonne, C., Joseph, P. \& Bocquet, L. 2007 Achieving large slip with superhydrophobic surfaces: Scaling laws for generic geometries. Phys. Fluids 19 (12), 123601. 